\title{
PolSAR Ship Detection Based on Neighborhood Polarimetric Covariance Matrix
}

\author{
Tao Liu, Ziyuan Yang, Armando Marino, Member, IEEE, Gui Gao, Member, IEEE and Jian Yang, Senior Member, IEEE
}

\begin{abstract}
The detection of small ships in polarimetric synthetic aperture radar (PolSAR) images is still a topic for further investigation. Recently, patch detection techniques, such as superpixel-level detection, have stimulated wide interest because they can use the information contained in similarities among neighboring pixels. In this paper, we propose a novel neighborhood polarimetric covariance matrix (NPCM) to detect small ships in PolSAR images, leading to a significant improvement in the separability between ship targets and sea clutter. The NPCM utilizes the spatial correlation between neighborhood pixels and maps the representation for a given pixel into a high-dimensional covariance matrix by embedding spatial and polarization information. Using the NPCM formalism, we apply a standard whitening filter, similar to the polarimetric whitening filter (PWF). We show how the inclusion of neighborhood information improves the performance compared with the traditional polarimetric covariance matrix. However, this is at the expense of a higher computation cost. The theory is validated via simulated and measured data under different sea states and using different radar platforms.
\end{abstract}

Index Terms-Constant false alarm rate (CFAR), Sea clutter, Statistical modeling, Synthetic aperture radar (SAR), Ship detection, Polarimetric Whitening Filter (PWF)

\section{INTRODUCTION}

$\mathrm{P}$ OLARIMETRIC Synthetic Aperture Radar (PolSAR) is a remote sensing system largely used for marine surveillance. Ship detection is an essential task for traffic surveillance, fishery monitoring, search and rescue, border control, environmental protection, etc. [1]. Generally, the backscattering signal from ships is stronger than the sea clutter. This leads to design detection algorithms based on the statistical analysis of SAR images [1]. These tests are generally focused on the clutter statistics, and therefore, they set the threshold adaptively based on the probability of false alarm (PFA) principle. The latter detection principle is named as the constant false alarm rate (CFAR) [1]. Up to now, the detection

This work was supported in part by the National Natural Science Foundation of China under Project 61771483, 41822105, 61490693, Key Research Plan of Hunan Province under Project 2019SK2173, the Field Foundation under Project 61404160109 and the Fundamental Research Funds for the Central Universities under Project 2682020ZT34.

T. Liu and Z. Yang are with the school of electronic engineering, Naval University of Engineering, Wuhan, 430033, China (e-mail: liutao1018@hotmail.com).

A. Marino is with the Faculty of Natural Sciences, University of Stirling, UK (armando.marino@stir.ac.uk).

G. Gao is with the Faculty of Geoscience and Environmental Engineering, Southwest Jiaotong University, Chengdu, 611756, China, and with the College of Traffic Engineering, Hunan University of Technology, Zhuzhou, 412007, China(e-mail: dellar@126.com).

J. Yang are with the department of electronic engineering, Tsinghua University, Beijing, 100084, China (e-mail:yangjian_ee@tsinghua.edu.cn). of small ships in PolSAR images is still an open issue. It has been proven that polarimetry can provide more information in detecting and classifying targets in SAR images, leading to the wide application of polarimetric SAR (PolSAR) to improve the performances for small ships. A review of detection techniques is presented in [2], and recent advancements are shown in [3].

The detectors using polarization can be divided into two categories: pixel-level detection and patch-level detection. Many traditional polarimetric detectors belong to pixel-level. Novak et al. proposed the optimal polarimetric detector (OPD) [4], which reaches the theoretical upper limit for polarimetric detectors (in case the assumption is met). Additionally, target decomposition algorithms were used for the polarimetric detector [5]-[11]. The Cameron decomposition method is applied by Ringrose et al. to detect ships from SIR-C data [5]. A polarization cross-entropy is proposed by Chen et al. for detecting ships [6]. The four-component model-based decomposition is also utilized for ship detection [7]. Moreover, polarization entropy is also introduced, derived from the Cloude-Pottier decomposition of the polarimetric covariance matrix [8]. An optimization method of the degree of polarization is proposed by Touzi et al. to enhance ship detection [9][10]. In addition, the symmetry detector uses the module of the correlation between co- and cross-channels because the sea clutter has reflection symmetry [11]. In the absence of ship statistics, the polarimetric whitening filter (PWF) proposed by Novak [12] utilizes the polarimetric information to minimize the speckle fluctuation. Its detection performance has been proved to be almost the same as that of the OPD [12]. To extend Novak's work to multi-look cases, Liu and Lopes et al. developed the MPWF [13]-[15]. Recently, Marino et al. proposed a polarimetric notch filter (PNF), which separates targets and sea clutter by minimizing the sea clutter power [16]-[17], which does not require prior information about the ships. Abundant experiments have validated its good performance [1][18]-[19].

In some studies, new ship detectors were proposed to utilize spatial correlation information at a patch level [20]-[22]. However, these methods were intensity-based detectors, and therefore, they do not take advantage of polarimetric information. The superpixel segmentation was proposed originally in computer vision [23]. Recently, it has been applied successfully to ship detection, wherein a ship can be viewed as one or several connected superpixels that can provide a more favorable structural information [24]. A superpixel-level CFAR detector has been proposed that uses weighted information entropy [25] [26]. Additionally, two superpixel-based detection methods using the difference of scattering mechanism are introduced in [27] and [28], respectively. He et al. proposed a novel automatic ship detection method, by considering several polarimetric dissimilarity measurements in the superpixel-level 
[29]. Cui et al. proposed a saliency detector for polarimetric SAR ship detection using a similarity test [30]. Another metric for ship detection is exploited based on the scattering similarity between ship and sea clutter [31]. As we know, superpixel-level detection can be divided into two stages. The first stage is the superpixel segmentation, and the second is feature extraction and detection. The first stage is crucial, and common segmentation is based on a likelihood ratio test, which can be expressed as a general trace form of the Wishart distance [30]. This is the same form as the PWF. In other words, the segmentation process can be seen as a ship detection exercise itself. Thus, by only considering the segmentation, spatial information is still not fully used. Lang et al. presented a ship detector in high-resolution SAR images by clustering pixel descriptors spatially with high dimensions [32], while the clustering only used the surrounding intensity ignoring the correlation among the pixels. Zhang et al. proposed an entirely different polarimetric matrix to detect targets obtained by a transformation by considering neighbor polarimetric covariance matrices [33][34]. However, the final matrix cannot be interpreted as a partial target since it lacks some properties, such as not being a positive semidefinite matrix. By combining deep learning techniques, Jin et al. obtained good results by proposing a patch-to-pixel convolutional neural network (P2P-CNN) for small ship detection in PolSAR images [35]. Additionally, Dai et al. used a convolutional neural network (CNN) to detect ships in a very complex environment [36]. However, explaining the $\mathrm{CNN}$ from a physical point of view is still difficult.

As both ships and sea clutter are spatially correlated, and ships will change the spatial correlation of surrounding clutter, we propose the spatial neighborhood polarimetric covariance matrix (NPCM). The NPCM combines the polarization and spatial characteristics expected to increase the separability between ship and sea clutter significantly. Briefly, the contributions of this paper can be divided into the following three aspects:

1) We proposed a novel polarimetric detector based on the spatial correlation of neighborhood pixels in PolSAR imagery. The neighborhood polarimetric covariance matrix (NPCM) was established. The method can be used with and without performing a pre-average (single look complex data, SLC, or multi-look complex data, MLC). The experiments showed that it returns a better performance compared with traditional detectors, which do not use spatial information.

2) The optimal size and therefore, the dimension of the NPCM was selected using the Akaike Information Criterion (AIC) and Bayesian Information Criterion (BIC) [45]. This ensures that the size is set to contain most of the correlation information in the NPCM.

3) To accelerate detection efficiency, a dimension reduction method for the NPCM was proposed. This was based on the principal component analysis (PCA) method [45]. In addition, the recursion method was adopted to calculate the NPCM for efficiency.

This paper is organized as follows. Section II presents the data forms in PolSAR imagery before establishing the neighborhood polarimetric covariance matrix (NPCM). Section III provides the methodology of the novel polarimetric detector and presents the main scientific problems and the corresponding solutions. In Section IV, the novel detector is validated by simulated and embedded data. In Section V, a description is provided on the measured SAR data that are used in the experiments, and finally, Section VI presents the main conclusions and perspectives.

\section{NeIGHBORHOOD POLARIMETRIC COVARIANCE MATRIX}

\section{A. Description of PolSAR Data}

Under the assumption of far field, the polarimetric scattering matrix can describe the scattering characteristics of a target [1][12]

$$
\mathbf{S}=\left[\begin{array}{ll}
S_{H H} & S_{H V} \\
S_{V H} & S_{V V}
\end{array}\right]
$$

where a linear Horizontal-Vertical basis is adopted. $S_{x y}$ represents the complex scattering coefficient. $y$ stands for the receiving polarization, and $x$ for the transmitting polarization. When the system is mono-static and satisfies the reciprocity assumption, $S_{H V}$ is approximately equal to $S_{V H}[12]$. The scattering vector is defined as follows:

$$
\mathbf{k}=\left[\begin{array}{l}
k_{1} \\
k_{2} \\
k_{3}
\end{array}\right]=\left[\begin{array}{c}
S_{H H} \\
\sqrt{2} S_{H V} \\
S_{V V}
\end{array}\right]
$$

To suppress speckle fluctuation in SAR images, polarimetric filtering or multi-look processing (box filtering) are generally used. A covariance matrix is obtained as follows [12][14].

$$
\mathbf{C}=\frac{1}{L} \sum_{i=1}^{L} \mathbf{k}_{i} \mathbf{k}_{i}^{\dagger}=\left[\begin{array}{ccc}
\left\langle\left. k_{1}\right|^{2}\right\rangle & \left\langle k_{1} k_{2}^{*}\right\rangle & \left\langle k_{1} k_{3}^{*}\right\rangle \\
\left\langle k_{2} k_{1}^{*}\right\rangle & \left\langle\left|k_{2}\right|^{2}\right\rangle & \left\langle k_{2} k_{3}^{*}\right\rangle \\
\left\langle k_{3} k_{1}^{*}\right\rangle & \left\langle k_{3} k_{2}^{*}\right\rangle & \left\langle\left|k_{3}\right|^{2}\right\rangle
\end{array}\right]
$$

where $L$ is the number of looks, $i$ is the $i t h$ pixel in multi-look proceeding pixels, the superscript $\dagger$ denotes conjugate transpose, $\langle$.$\rangle is the finite averaging operator by L$, and $\|$ is the operator of absolute value. The dimension of the polarimetric covariance matrix is $d \times d$, and $d$ is the dimension of the scattering vector $\mathbf{k}$.

It is well known that speckle is an inherent feature of synthetic aperture radar (SAR) images [12][14]. The polarimetric whitening filter is originally proposed (PWF) to optimally suppress speckle in polarimetric SAR imagery [12]. Later Novak et al. [12] showed that the PWF enhances target detection performance. Lopes and Liu et al. further developed the PWF to the multi-look polarimetric whitening filter (MPWF) [13]-[14], where a polarimetric covariance matrix represents SAR data.

\section{B. Neighborhood Polarimetric Covariance Matrix}

A pixel can be characterized by one scattering matrix S, and traditionally a pixel patch is characterized by a polarimetric covariance matrix $\mathbf{C}$. Although $\mathbf{C}$ can depict the second order statistics of a patch of pixels, it cannot easily express the spatial correlation of these pixels seen as separate targets. One 
example of the algorithms that attempts to evaluate the correlation of neighbor pixels is the Cloude-Pottier decomposition with the spectral analysis of $\mathbf{C}$ [37]. However, the diagonalization has the drawback that is constrained to monitor a maximum of 3 targets which needs to be orthogonal. Nevertheless, the entropy has been widely used for ship detection [9][10].

However, it is expected that a pixel of the sea would be correlated with the surrounding pixels, whether it is made of sea clutter or ships.

The correlation of a pixel with its neighbor can be represented using seven types of local neighborhood pixel collections: horizontal, vertical, diagonal, anti-diagonal, horizontal-vertical, diagonal-anti-diagonal, and square. As shown in Fig. 1, these operations can be performed using $\omega \times \omega$ windows ( $\omega$ is the size of window). The collection is referred to in terms of the central pixel. The pixels can be represented as a vector with dimensionality $n d \times 1$, where $n$ is the number of the neighborhood pixels.
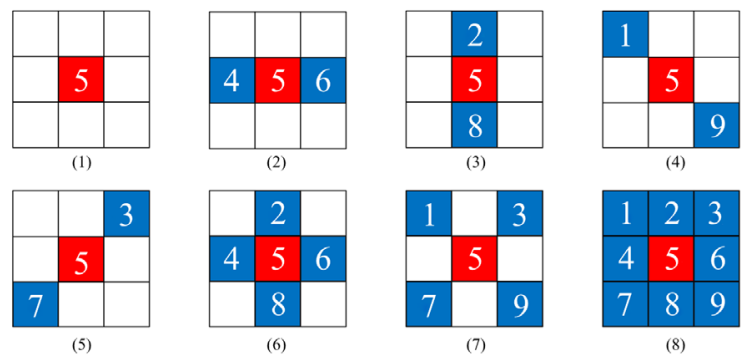

Fig 1. Seven types of local neighborhood collections: (1) center pixel (2) horizontal, (3) vertical, (4) diagonal, (5) anti-diagonal, (6) horizontal-vertical, (7) diagonal-anti-diagonal, and (8) square

As shown in Fig. 1, to describe the center pixel (red one) in a $3 \times 3$ region, we could use the following spatial correlation vectors according to different neighborhood types:

1) Horizontal: $\mathbf{p}=\left[\mathbf{k}_{4}, \mathbf{k}_{5}, \mathbf{k}_{6}\right]^{T}$

2) Vertical: $\mathbf{p}=\left[\mathbf{k}_{2}, \mathbf{k}_{5}, \mathbf{k}_{8}\right]^{T}$

3) Diagonal: $\mathbf{p}=\left[\mathbf{k}_{1}, \mathbf{k}_{5}, \mathbf{k}_{9}\right]^{T}$

4) Anti-diagonal: $\mathbf{p}=\left[\mathbf{k}_{3}, \mathbf{k}_{5}, \mathbf{k}_{7}\right]^{T}$

5) horizontal-vertical: $\mathbf{p}=\left[\mathbf{k}_{2}, \mathbf{k}_{4}, \mathbf{k}_{5}, \mathbf{k}_{6}, \mathbf{k}_{8}\right]^{T}$

6) diagonal-anti-diagonal: $\mathbf{p}=\left[\mathbf{k}_{1}, \mathbf{k}_{3}, \mathbf{k}_{5}, \mathbf{k}_{7}, \mathbf{k}_{9}\right]^{T}$

7) Square: $\mathbf{p}=\left[\mathbf{k}_{1}, \mathbf{k}_{2}, \mathbf{k}_{3}, \mathbf{k}_{4}, \mathbf{k}_{5}, \mathbf{k}_{6}, \mathbf{k}_{7}, \mathbf{k}_{8}, \mathbf{k}_{9}\right]^{T}$,

where $\mathbf{k}_{i}$ denotes the scattering vector of the corresponding pixel $i, n$ is the number of the chosen pixels, $d$ is the dimension of the scattering vector $\mathbf{k}$, and $T$ denotes transpose. In this way, the representation of an individual pixel is established from the 3D polarimetric space to a higher-dimensional correlation space, and both the spatial and polarimetric information is contained.

The scattering vector is assumed to be a circular Gaussian distribution with zero mean in the complex plane; therefore, the neighborhood polarimetric scattering vector will still be composed of Gaussian processes. The statistics of the new vector depends only on the covariance matrix due to the zero-mean hypothesis. The neighborhood vector's covariance matrix is named as neighborhood polarimetric covariance matrix (NPCM) and can be calculated as follows:

$$
\mathbf{C}=\frac{1}{L} \sum_{i=1}^{L} \mathbf{p}_{i} \mathbf{p}_{i}^{\dagger}
$$

where $L$ is the number of looks, $i$ is the $i t h$ pixel, and the superscript $\uparrow$ denotes conjugate transpose. The dimension of the NPCM $\mathbf{C}$ is $n d \times n d$.

The PDF of the NPCM is Wishart distributed based on multivariate complex Gaussian distribution, which can be used to describe fully developed speckle [38][39]

$$
f_{C}(\mathbf{C})=\frac{L^{L d}|\mathbf{C}|^{L-d} \exp \left(-L \operatorname{Tr}\left(\boldsymbol{\Sigma}^{-1} \mathbf{C}\right)\right)}{\Gamma_{d}(L)|\mathbf{\Sigma}|^{L}}
$$

where $L$ is the number of looks, $d$ is the dimension of $\mathbf{C}$, $\operatorname{Tr}(\bullet)$ is the trace operator, $\Gamma(\bullet)$ denotes gamma function and $\Gamma_{d}(L)$ is:

$$
\Gamma_{d}(L)=\pi^{\frac{1}{2} d(d-1)} \Gamma(L) \cdots \Gamma(L-d+1)
$$

$\mathbf{\Sigma}=\mathrm{E}\{\mathbf{C}\}$ is the neighborhood covariance matrix of the new vector in the Gaussian case, and $\mathrm{E}(\bullet)$ is the expectation operator. If the texture is not constant in practice, the multivariate product model can be used to model more complicated statistics of the sea clutter [41].

\section{NOVEl NeIGHBORHOOD DETECTOR}

In this section, the methodology is analyzed, and the standard whitening filter (SWF) is chosen to produce the final distance used for detection. This is due to it having outstanding performances in the absence of ship statistics. Finally, a dimension reduction method is discussed to accelerate the calculation, which may cause some loss of the detection performance.

\section{A. Methodology}

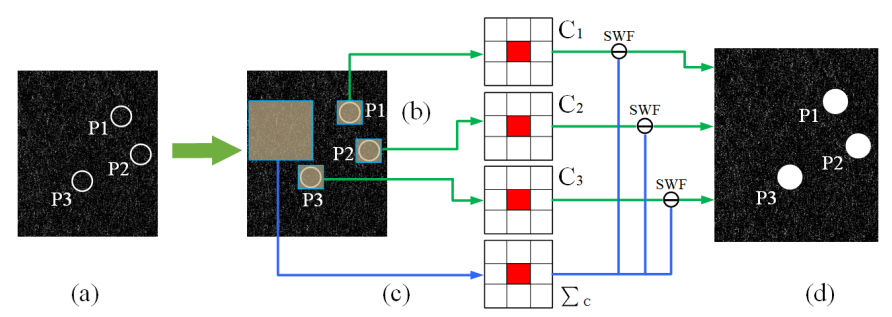

Fig. 2. Flowchart of the proposed ship detection strategy.

The flowchart of the new ship detection algorithm is shown in Fig. 2. Starting from a PolSAR image [Fig. 2(a)], we can consider pixels such as $\mathrm{P} 1, \mathrm{P} 2$, and $\mathrm{P} 3$. The polarimetric scattering vectors of the neighborhood pixels are chained together and form a high dimensional vector for each centered pixel [Fig. 2(b), green lines] according to the neighborhood definition in Fig 1. The outer product then calculates the NPCM 
for each feature vector built by the chained neighborhood pixels. At this stage, the reference of the NPCM for the sea clutter is evaluated by using sliding windows or selecting a pure clutter region close to the area to be detected [Fig. 2(c), blue line]. Finally, a standard whitening filter (SWF, similar to the PWF in terms of processing) is used to derive the operator that would be used for ship detection [Fig. 2(d)].

The SWF can be seen as an extension of the PWF when considering neighborhood pixels. The output of the SWF is as follows [12]:

$$
z=\frac{1}{L} \sum_{i=1}^{L} \mathbf{p}_{i}^{\dagger} \boldsymbol{\Sigma}^{-1} \mathbf{p}_{i}=\operatorname{tr}\left(\boldsymbol{\Sigma}^{-1} \mathbf{C}\right)
$$

where $\operatorname{tr}(\cdot)$ is the trace operator and $L$ is the number of independent samples (or looks), which is consistent with that in Eq (5). According to the literature [40], $z$ obeys a Gamma distribution in the Wishart assumption:

$$
z \sim \gamma\left(L d, \frac{1}{L}\right)
$$

where $\gamma(\alpha, \beta)$ denotes Gamma distribution of which the shape parameter is $\alpha$, and scale parameter is $\beta . \gamma(\alpha, \beta)$ can be expanded as [40]

$$
\gamma(\alpha, \beta)=\frac{1}{\Gamma(\alpha)} \frac{1}{\beta}\left(\frac{x}{\beta}\right)^{\alpha-1} e^{-\frac{x}{\beta}}
$$

Eq. (8) gives the statistics of the SWF in the Wishart distribution case [40]. In this statistical model, the PFA can be derived from [41]:

$$
P_{f a}=\frac{\Gamma(d L, L T)}{\Gamma(d L)}
$$

where $P_{f a}$ is the false alarm rate, and $d$ is the dimension of the scattering vector. The incomplete gamma function and the gamma function are presented as follows [40]

$$
\Gamma(x)=\int_{0}^{\infty} e^{-t} t^{x-1} d t, \Gamma(a, x)=\int_{x}^{\infty} e^{-t} t^{a-1} d t
$$

With the increasing SAR resolution and the roughness of sea conditions, the Wishart assumption may not hold [41]. In [41] it was found that the gamma distribution is a special case of the generalized gamma distribution ( GГD). The GГD can also be adopted to model the SWF output. Therefore, we get

$$
\gamma(\alpha, \beta)=f(x ; \alpha, 1, \alpha \beta)
$$

where $f$ is the generalized gamma distribution (GGD) as follows:

$$
f(z ; k, v, \sigma)=\frac{|v| k^{k}}{\sigma \Gamma(k)}\left(\frac{z}{\sigma}\right)^{k v-1} \exp \left\{-k\left(\frac{z}{\sigma}\right)^{v}\right\}
$$

$k, \nu, \sigma$ are the shape, power, and scale parameters, respectively.

The PFA/PD can be obtained as follow:

$$
P_{f a / d}=\int_{T}^{\infty} f(z ; k, v, \sigma) d z=\left\{\begin{array}{c}
1-\Gamma\left[k, \eta T^{v}\right], v>0 \\
\Gamma\left[k, \eta T^{v}\right], v<0
\end{array}\right.
$$

The threshold can be easily derived

$$
T=\left\{\begin{array}{l}
\left(\frac{1}{\eta} \Gamma^{-1}\left[k, 1-P_{f a}\right]\right)^{1 / v} \\
\left(\frac{1}{\eta} \Gamma^{-1}\left[k, P_{f a}\right]\right)^{1 / v}
\end{array}\right.
$$

where $\eta=k \sigma^{-v}$. The calculation of the threshold is analytical.

Since the vectors used to form the NPCM matrix have the same underlying distribution model, in this paper we use GГD to calculate the analytical threshold of the CFAR on the whitening of the NPCM.

\section{B. Size Determination of the Neighborhood window}

The size (i.e., dimension) of the NPCM will greatly affect the detection performance and the calculation complexity. It is important to determine the sound size for the NPCM. The intensity auto-covariance of SAR image was evaluated in [42][43]:

$$
C_{F}(\Delta x, \Delta y ; L)=\frac{1}{L}\left[\sin c^{2}\left(\frac{\Delta x}{r_{x}}\right) \sin c^{2}\left(\frac{\Delta y}{r_{y}}\right)\right]
$$

where $r_{x}$ and $r_{y}$ are the spatial resolutions of a SAR system.

$x$ and $y$ means the imaging directions in the image plane.

It can be noticed that when the spatial distance becomes larger, the correlation rapidly turns weaker. Therefore, it may be more appropriate to keep the correlation coefficients limited to a small distance. Here, we adopt the approach of truncating the auto-covariance. To simplify the calculation, we use a one-dimensional auto-covariance first, where the correlation function is

$$
C_{F}(\Delta x ; L)=\frac{1}{L} \sin c^{2}\left(\frac{\Delta x}{r_{x}}\right)
$$

We can measure all the correlation coefficients for all directions using this (including horizontal, vertical, diagonal, anti-diagonal, etc.).

The size of the neighborhood matrix is determined by the Akaike Information Criterion (AIC) and Bayesian Information Criterion (BIC). Both can be successfully used in auto regression (AR) models [44]. We found out that to not have negative effects by limited resolution, the size of the NPCM should be $3 \times 3$ or $5 \times 5$. However, for the high-resolution radar, the size may be larger, which will also return a substantial increase in calculation complexity.

\section{Dimension Reduction}

Since the dimension of the NPCM is generally large, the computation complexity may be problematic. We, therefore, implemented a dimension reduction for the NPCM detector. Even when the size of the NPCM search space is $3 \times 3$, the dimension of the NPCM is $9 d \times 9 d$ (when the dimension of the scattering vector is $d=3$, the dimension of the NPCM is $27 \times 27$ ). The square form of the NPCM makes the computation very complex. Therefore, we consider proposing a dimension reduced detector based on the NPCM, as shown in Fig 3.

We perform a principal component analysis (PCA) to reduce the dimension of the NPCM matrix. PCA is largely used as a 
tool in exploratory data analysis and for building predictive models. It is often used to visualize genetic distances and relationships between populations. PCA is the simplest of the true eigenvector-based multivariate analyses, and in some circumstances, its operation can be thought of as revealing the internal structure of the data in a way that best explains data variance. Additionally, PCA is widely used in space-time adaptive processing (STAP) [45].

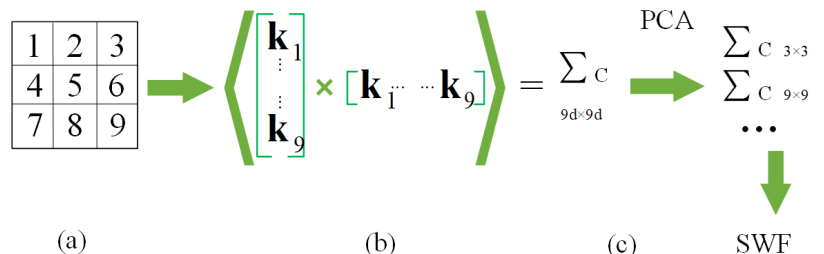
(d)

g. 3. Flowchart of dimension reduction by PCA.

The dimension of the $9 d$-dimension polarimetric channels can be reduced. In this study, we decided to use 9 or 3 dimensions, and the covariance matrix turns to $9 \times 9$ or $3 \times 3$. This is because the computation complexity of $9 \times 9$ is similar to the simplest types of NPCM (Horizontal or Vertical).

Because the inverse matrix of NPCM is calculated, the corresponding eigenvectors should be chosen by the 9 or 3 smallest eigenvalues. The final step of whitening is still performed on the matrix with reduced dimensions [Fig.3(f)].

Interestingly, we can see the NPCM as a generalization of the ordinary polarimetric matrix [33]. This allows us to perform a fair comparison of these different pixel arrangements in simulated or real data.

\section{Extension of the NPCM to data provided in covariance matrix format}

The NPCM can also be evaluated when data is provided in a covariance matrix or MLC (Multi Look Complex) format instead of the SLC. This is sometimes used by data providers to reduce the size of data stored.

To extend the detector to those cases, we use the vectorization of the polarimetric covariance matrix as the new scattering vector to establish the spatial correlation. The scattering vector is defined from the covariance matrix $\mathbf{C}$, expressed as follows when the polarimetric matrix is three dimensional $(d=3)[46]$

$$
\begin{aligned}
& \mathbf{t}=\left[t_{1}, t_{2}, t_{3}, t_{4}, t_{5}, t_{6}\right] \\
& =\left[\left(\left\langle\left. k_{1}\right|^{2}\right\rangle\right)^{1 / 2},\left(\left\langle\left|k_{2}\right|^{2}\right\rangle\right)^{1 / 2},\left(\left\langle\left|k_{3}\right|^{2}\right\rangle\right)^{1 / 2},\right. \\
& \left.\left(\left\langle k_{1}^{*} k_{2}\right\rangle\right)^{1 / 2},\left(\left\langle k_{1}^{*} k_{3}\right\rangle\right)^{1 / 2},\left(\left\langle k_{2}^{*} k_{3}\right\rangle\right)^{1 / 2}\right]
\end{aligned}
$$

where $\mathbf{t}$ is a 6-D complex vector, * is the superscript of conjugation. The NPCM of partial vectors is established as follows

$$
\mathbf{C}=\frac{1}{L} \sum_{i=1}^{L} \mathbf{p}_{i} \mathbf{p}_{i}^{\dagger}
$$

When we use quad-pol $(d=3)$, the dimension of the new $\mathrm{NPCM} \mathbf{C}$ is $54 \times 54$. It should be noted that the processing with $\mathbf{t}$ is equivalent to using $\mathbf{k}$, except for the increased number of dimensions.

\section{Detection Performance with Simulated Data}

This section presents experimental results to evaluate the performance of the novel detectors for the SLC dataset. The MLC data results are similar; therefore, they are not presented here and are discussed only in the measured data section. We compare the performance of the detector using patch simulations.

\section{A. Generation of Simulated Data}

We suppose the size of the spatial operator to be $3 \times 3$, and the polarimetric scattering vector is $3 \times 1$. Therefore, the neighborhood scattering vector is $27 \times 1$ and the NPCM is $27 \times 27$. The Wishart distribution covariance matrices with the dimensionality $27 \times 27$ are generated. The Wishart distribution was colored by an asymptotic matrix obtained by averaging a large homogeneous region of sea in real SAR data from RADARSAT2. The measured data are described in detail in Section V.

The patches of SAR images are generated by Monte Carlo simulation. In the generation of the simulated NPCM for both ships and clutter, the number of looks was set to $L=1$. Each of the matrices must be ranked $1(\mathrm{~L}=1)$ because it represents a single pixel in a single look complex (SLC) case. The synthetic data set consists of $N=1,000,000$ covariance matrix samples drawn from a complex, circular, and zero-mean Wishart distribution.

\section{B. Recursion Calculation}

Even though the neighborhood window size is $3 \times 3$, calculations of the NPCM for each pixel are complex. There are many repetitive computations between the NPCMs for adjacent pixels. Therefore, we proposed a refined algorithm to calculate the NPCM to merge the repetitive computations. It will not alter the result as long as the calculation accuracy meets the requirement.

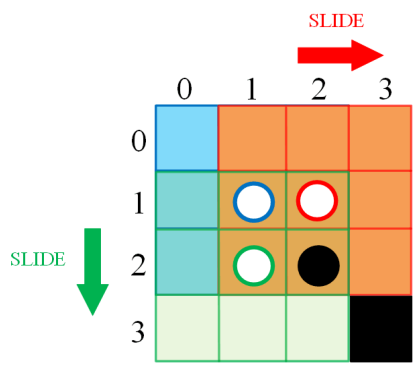

Fig. 4. Illustration of recursion of the NPCM calculation.

We suppose the NPCM is calculated row by row. Because the NPCM is calculated by the neighborhood pixels, we define the beginning row and column number as zero in the original image, shown in Fig 4. In the first row of the NPCM, we can see when the pixel moves to the next neighbor pixel (from the blue circle to the red circle), three pixels surrounding the red circle (the orange color pixels) should be added to calculate the NPCM. So does the first column. From the beginning of the second row or column (the green circle to the black real circle), 
only one more pixel (black color) should be added to calculate the NPCM when calculating the neighbor pixel (black circle). This recursion method avoids the repeated calculation of correlations between the neighborhood pixels.

From the illustration in Fig 4, we can see the repetitive computations are all deleted, and the computation was reduced to $1 / 9$ of the original case except the first row and column. If the size of the neighborhood window is $5 \times 5$, the computation is reduced to $1 / 25$.

\section{Comparisons between Pixel and Patch Detectors}

Since the polarimetric whitening filter (PWF) gives the closest performance to the optimal polarimetric filter (OPD) when prior information of targets are missing [4], the PWF is selected as the pixel detector for the comparison. Also, seven types of neighborhood windows are introduced (as in Fig 1) and divided into three types: 3 pixels, 5 pixels, and 9 pixels. It can be expected that the same pixel number could provide similar detection performance. Here Horizontal, Horizontal-Vertical, and Square neighborhood windows are selected to represent the 3,5,9 pixels, respectively.

In this section, the Receiver Operating Characteristic (ROC) is used to assess performance. The probability of false alarm is defined as

$$
P_{f a}=\frac{n_{f a}}{n},
$$

where $n_{f a}$ is the number of false alarms and $n$ is the total number of clutter samples. The probability of detection is denoted as

$$
P_{d}=\frac{n_{d}}{n_{t}}
$$

where $n_{d}$ is the number of the detected targets and $n_{t}$ is the total number of targets. By forming the ROC curves, the threshold (and therefore $P_{f a}$ ) is varied and the values of $P_{d}$ and $P_{f a}$ are recorded and plotted [47]. In this section, Monte Carlo simulations are used to draw the ROC curves. The total number of simulations is $n_{t}=n=1,000,000$. The quantization of the thresholds for the detection is composed of 1,000 equal interval values that produce very small estimation problems. Here, TCR is defined as [4]

$$
T C R=\operatorname{tr}\left(\boldsymbol{\Sigma}_{T}-\boldsymbol{\Sigma}_{C}\right) / \operatorname{tr}\left(\boldsymbol{\Sigma}_{C}\right),
$$

where $\boldsymbol{\Sigma}_{T}, \quad \boldsymbol{\Sigma}_{C}$ are the estimated polarimetric covariance matrices of targets and clutter, respectively.

The results are presented in Fig 5. "PWF" denotes the PWF detector, "NPCM-SQ" denotes the square neighborhood detector, "NPCM-A" represents the Horizontal neighborhood detector (A means "across"). "NPCM-AS" is the Horizontal-Vertical neighborhood detector (S means "standing"), and "NPCM-3D", "NPCM-9D" are the detectors starting from the square neighborhood after a PCA reducing to $3(3 \times 3)$ or $9(9 \times 9)$ final dimensions. Here the $\mathrm{H}$ and $\mathrm{V}$ are not used to avoid confusion with polarization channels. The results show that the NPCM-SQ gives the best performance, followed by the "NPCM-AS," especially when the TCR is large enough. The standard PWF gives the worst performance. The "NPCM-AS" and "NPCM-9D" provide almost the same performance, which is much better than "NPCM-A." Though the dimension of "NPCM-9D" is the same as that of "NPCM-A," the "NPCM-9D" considers all the square pixels before applying the PCA to improve performance. The "NPCM-3D" is better than the PWF though they have the same dimension. We can see the more significant the dimension of NPCM is, the better the performance is. Also, the PCA method can reach high performance second only to the full square NPCM.

\section{Assessment of Different Detectors via Embedded Data}

Since the clutter spatial correlation is difficult to be simulated, we assess performance by using a clutter patch from measured data. We extract a pure clutter area in the measured SAR data and then embed the targets to construct a detection scene. Here the number of ships is 50, and the TCR is between 0 and 1 when uniformly distributed.

The size of the pure clutter region is $1207 \times 964$, which is randomly selected from the measured data. The detection results are presented in Fig. 6. A green rectangle identifies detected ships, a red rectangle shows omitted ships, and a yellow rectangle represents false alarms.

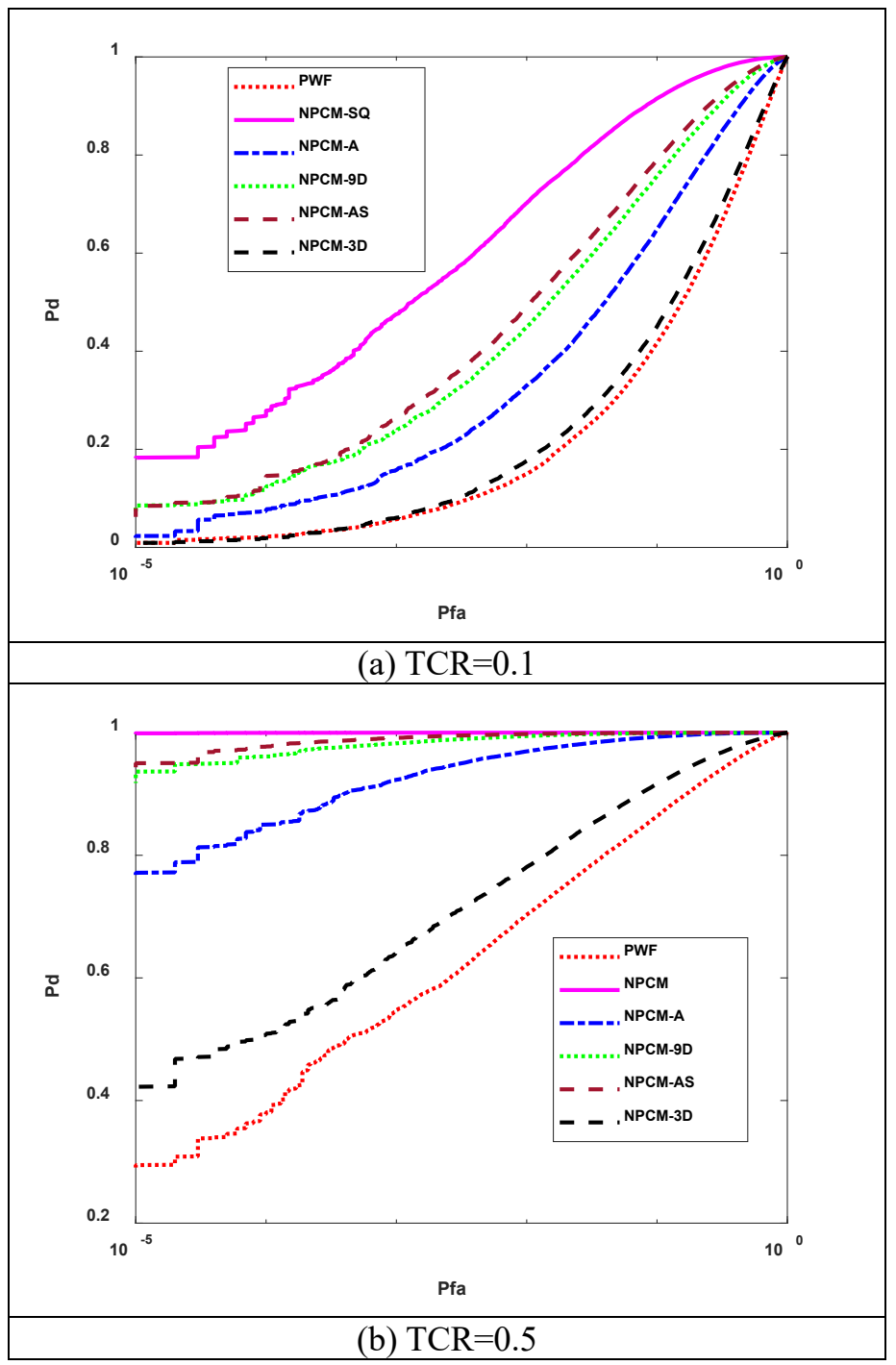




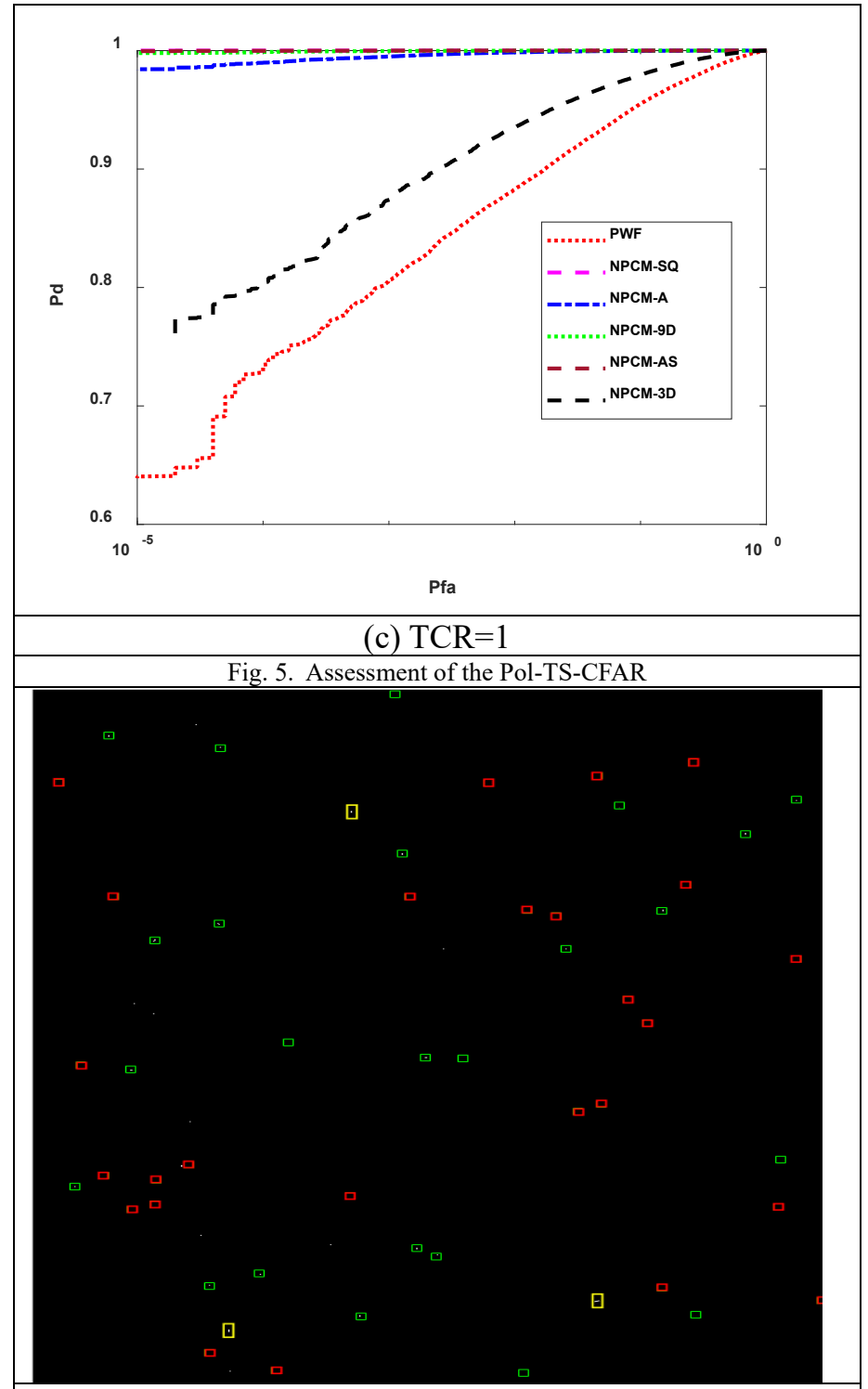

(a) PWF

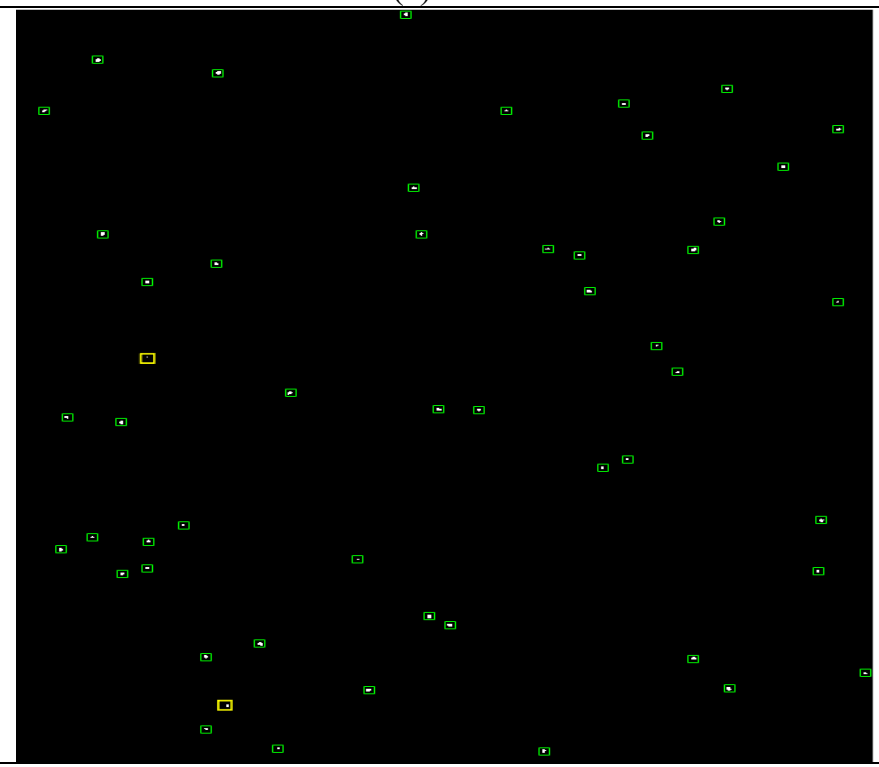

(b) NPCM-SQ

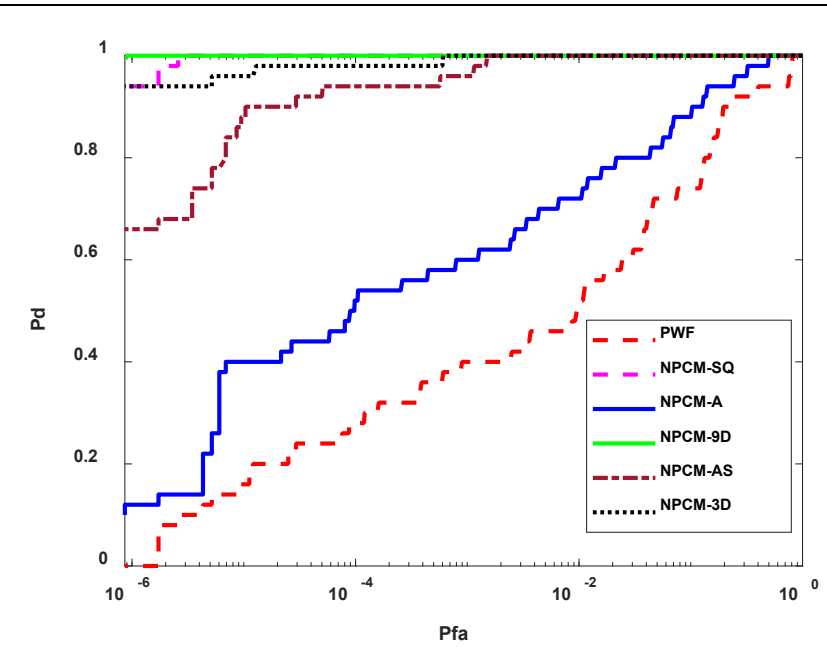

Fig. 7. Assessment of the detectors with 50 ships by ROC

The ROC of the embedded data is presented in Fig 7, showing that the NPCM gives the best performance. As expected, the larger the dimension of the neighborhood pixels, the better the detection performance. Here the "NPCM-3D" based on PCA reaches a perfect performance, even better than the "NPCM-AS" without reduction. The "NPCM-9D" based on PCA reaches a perfect performance, which is better than the full square version without reduction.

\section{Detection Performance with Measured Data}

The novel detectors are validated in this section via measured data. The formats of the measured data include a single look complex (SLC) and a multi-look complex (MLC) according to the data type of the data source.

\section{A. Dataset Details}

Two datasets are used here. The first dataset is the fully polarized RADARSAT-2 dataset. This high-quality, fully polarized image was taken by radar satellite in the North Sea in November 2013, where automatic identification system (AIS) information was also collected [18]. The beam is FQ12, and the angle of incidence is approximately $32^{\circ}$. The chirp bandwidth is $30 \mathrm{GHz}$ with the central frequency at C-band $(5.4 \mathrm{GHz})$. Scenes in this dataset are in SLC format, where the background covers approximately $25 \times 25 \mathrm{~km}$, with a slant range resolution of $5.2 \mathrm{~m}$, and an azimuth resolution of $7.6 \mathrm{~m}$. The noise equivalent sigma zero (NESZ) of the SAR image is about -36 $\mathrm{db}$. More information about this dataset can be found in [1]. The position information of the ship Automatic Identification System (AIS) system will be used to verify the accuracy of the experiment. In this case, there are 11 ships with AIS.

The other dataset is obtained using the NASA/JPL AIRSAR, installed on a DC-8 aircraft during the PACRIM-2 mission [48]. The dataset acquired on 4 October 2000 is also fully polarimetric. The SAR image was taken in the inland waters of Kojima Bay in Japan. The dataset is stored in MLC format, with the multi-look being $1 \times 9$. The slant range resolution is $3 \mathrm{~m}$, and the azimuth resolution is $4 \mathrm{~m}$. More information and details of the dataset can be found in [48]. L (1.25 GHz) and C (5.36 GHz) band data are included in this dataset, where $\mathrm{L}$ band is used to detect ships and $\mathrm{C}$ band to retrieve the wind speed. 
The meteomarin of the real datasets are presented here. The wind speed in Radarsat 2 is 32 knots in the RADARSAT-2 dataset, which is a gale. The sea state belongs to the very rough state. Since the cross-polarization relationship is not affected by incident angle and wind direction, we use cross-polarization to estimate wind speed, simplifying the process of retrieving wind speed from SAR image at sufficiently high wind speeds [49]. The result of retrieved wind speed is approximately $16 \mathrm{~m} / \mathrm{s}$, consistent with the value in [49]. In the second dataset, the averaged wind speed is also estimated by the cross-pol relationship, which is $12.5 \mathrm{~m} / \mathrm{s}$. This shows the sea state to be moderate to high.

\section{B. Size Analysis of NPCM Window}

To assess the best dimension of the spatial windows, we calculated the spatial auto-correlations of intensity pixels for clutter and targets in RADARSAT2, as shown in Fig 8. The MATLAB codes of the spatial correlation calculation are from https://github.com/ahmetcecen/MATLAB-Spatial-CorrelationToolbox.

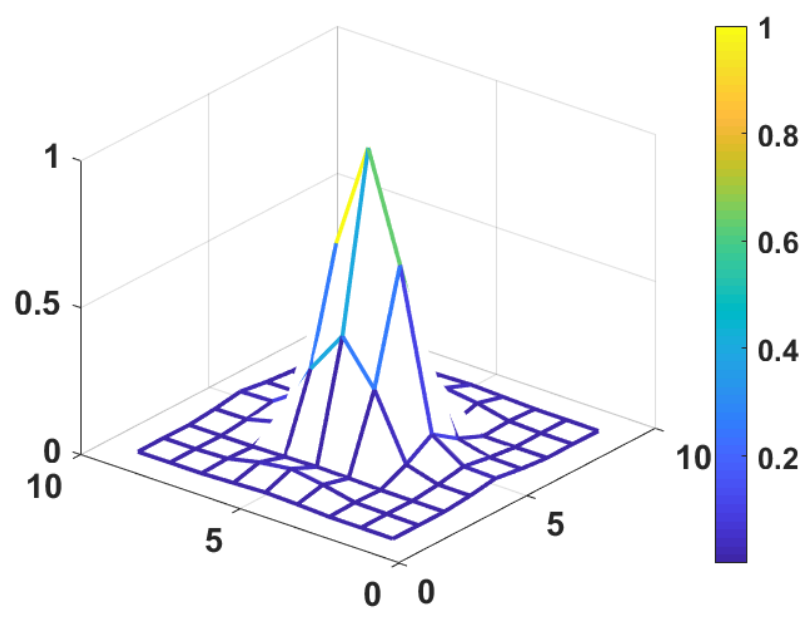

(a) S11-clutter spatial correlation function

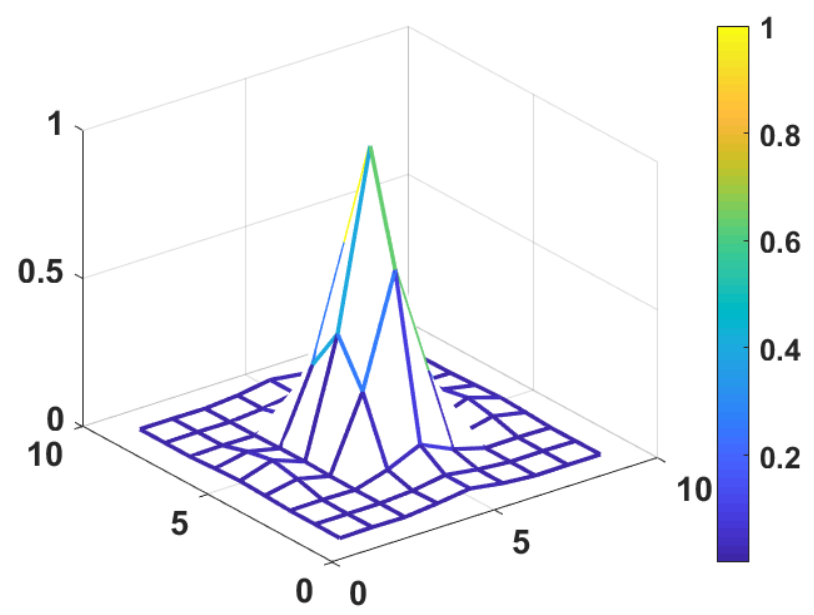

(b) S12-clutter spatial correlation function

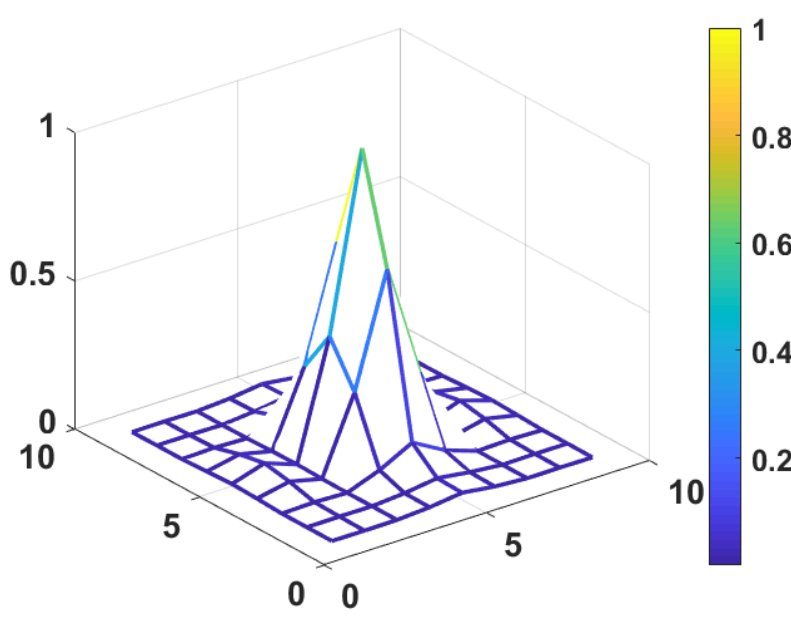

(c) S22-clutter spatial correlation function

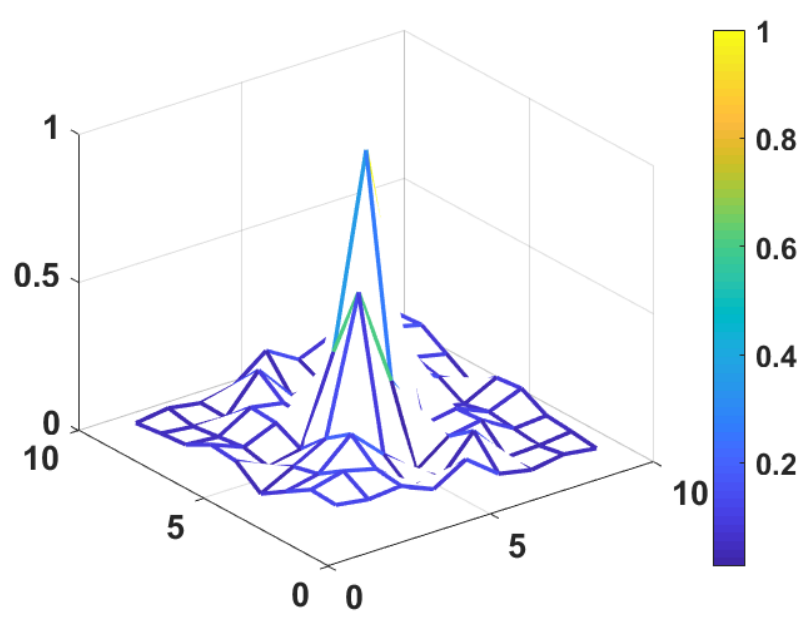

(d) S11-Target spatial correlation function

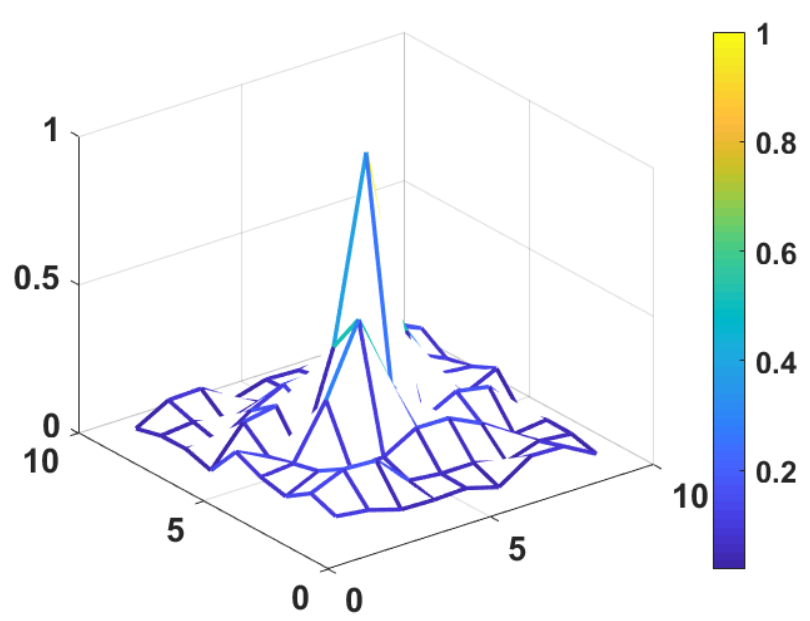

(e) S12-Target spatial correlation function 


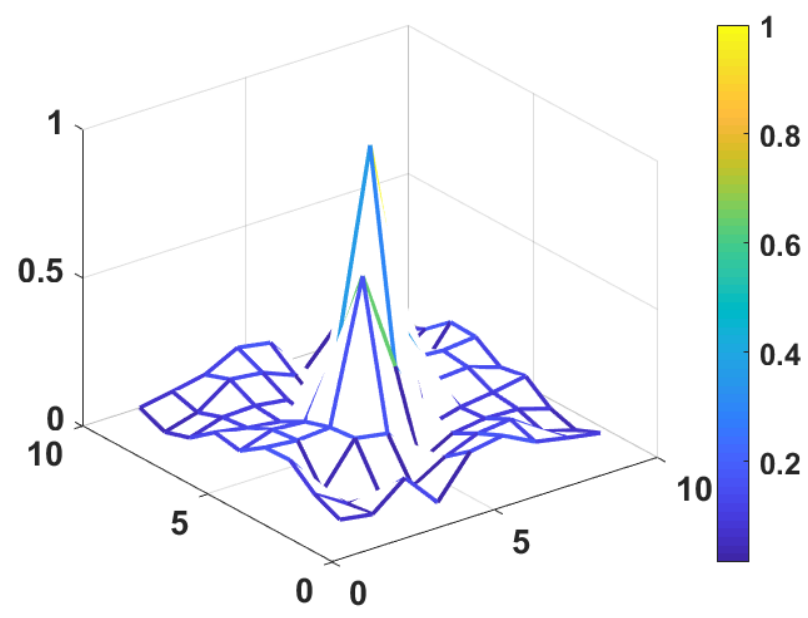

(f) S22-Target spatial correlation function

Fig. 8. Spatial correlation functions of different polarimetric channels involving targets and clutter

In the figures, the central pixel is at coordinate (5,5). After calculating the $\mathrm{AIC}$ and $\mathrm{BIC}$, we evaluated that the mean order in each direction is 1 , showing that only one extra pixel in each direction was adding significant information. Therefore, we decide to select the searching window $3 \times 3$ for the spatial operators. If the radar resolution is higher, the size of the spatial operator can be increased. Similar results are obtained with AIRSAR imagery.

\section{Goodness-of-Fit Test of the Statistical models}

This section tested whether the Gamma model or the generalized gamma model (GGD) could be used as a statistical model for real data after we apply the NPCM whitening. The selected area A is used to test the goodness-of-fit to the statistical models of sea clutter in the RadarSat-2 imagery. There are 11 ships in the RADARSAT2 data, labeled labelled $\mathrm{S} 1$ to $\mathrm{S} 11$. The selected area A is shown in Fig. 9, with a size of $1207 \times 964$. The PDFs of different statistical models are shown in Fig. 10. The real data are shown in black, the gamma model in green, and the GGD is shown in blue. The results show that GGD can perfectly fit the sea clutter. Moreover, the Kullback-Leibler (KL) distance [50] is used to measure the goodness-of-fit with values where there are 3.8103e-04 for the GGD model, consistent with Fig. 10.

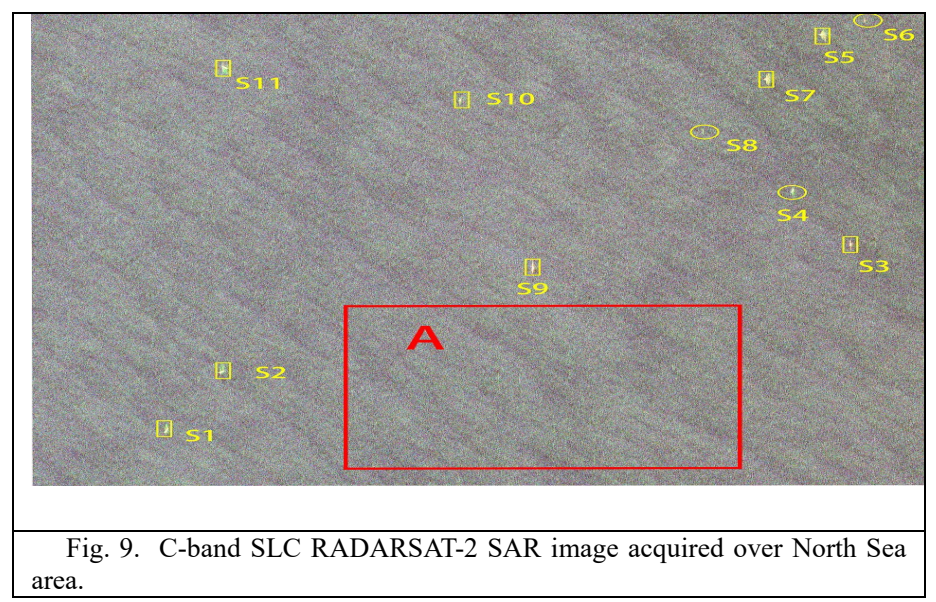

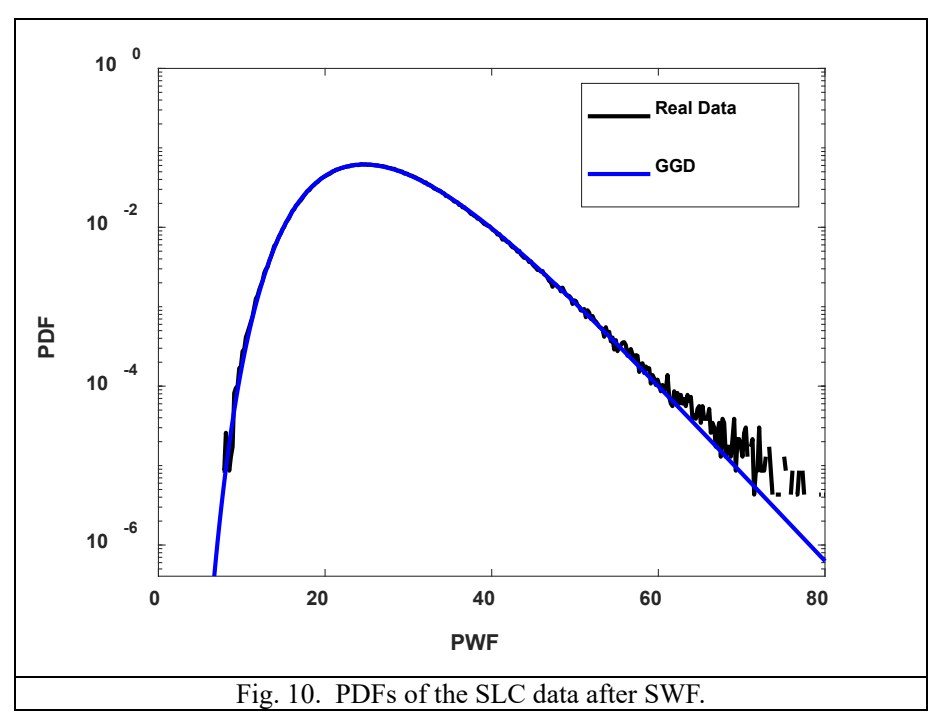

As is shown in Fig 11, the AIRSAR dataset consists of 21 targets, and the size of the selected clutter area B is $231 * 611$. The PDFs of different statistical models of the sea clutter are presented in Fig 12. The KL distance is 0.0022 for the GGD model, showing that the GGD can model the sea clutter quite well.
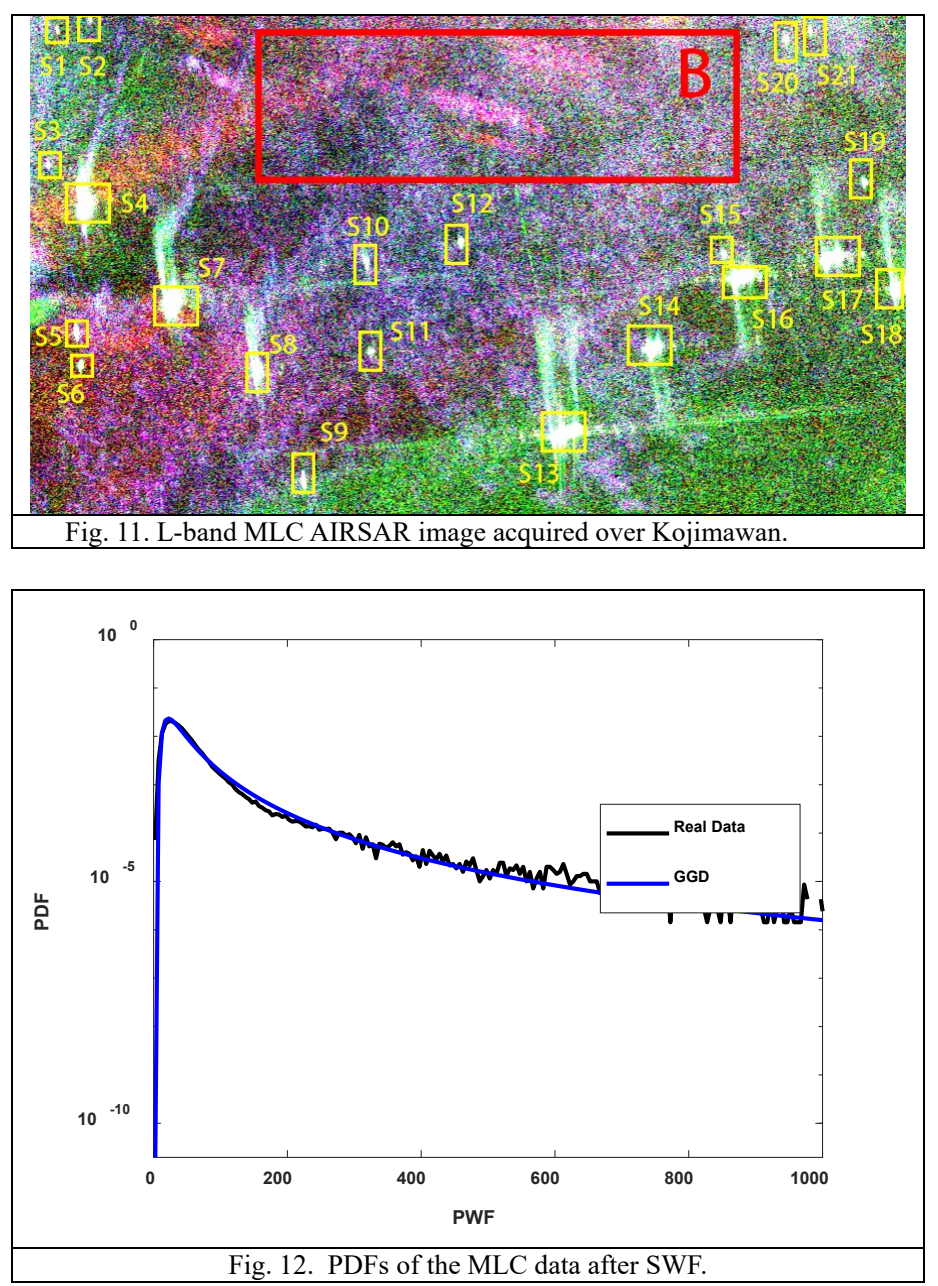


\section{Detection Flow of the Block CFAR Algorithm}

A CFAR framework called fast block CFAR is used to perform the detection [51]. Firstly, the region of interest (ROI) is divided into equally sized blocks depending on the resolution. Secondly, PWF is performed in each block, and the brightest pixels of each patch are truncated by a simple threshold so that the remaining pixels belong exclusively to the sea surface. Thirdly, an estimation of the neighborhood polarimetric covariance matrix for sea clutter is performed. Finally, with the estimated NPCM of sea clutter in every patch, the different dimensional whitening filters are performed, and a decision threshold inherent to the patch under test is set. Thus, threshold selection is adaptive to the image patch, providing some robustness against changes in clutter. If the ROI is not large enough or the sea is homogeneous, we can also select a small homogeneous area and estimate the clutter NPCM to be the averaged NPCM for the whole ROI.

\section{Performance Assessment}

Three indexes are used to assess the performance of different detectors in two datasets: 1) the false alarm rate maintenance, 2) the figure of merit (FOM), and 3) the ROC curves.

The CFAR loss is defined as follows [41]

$$
C_{L}=\left|20 \log \left(\bar{P}_{f a} / \widehat{P}_{f a}\right)\right|
$$

$C_{L}$ is a function indicating the distance between the pre-determined PFA $\bar{P}_{f a}$ and the measured PFA $\widehat{P}_{f a}$. The CFAR loss is used to assess the accuracy of the statistical model, but it cannot be used to evaluate the ship detection performance.

For more complicated scenes, the FOM is a suitable index for performance evaluation when the actual Pfa is constant for different detectors. FOM is defined as in [48]

$$
F o M=\frac{N_{t d}}{\left(N_{f a}+N_{g t}\right)}
$$

where $N_{t d}$ is the number of targets that are detected, $N_{f a}$ is the number of false alarms, $N_{g t}$ is the total number of targets.

In our performance assessment, we added three polarimetric detectors to be compared. The first is the well-known PWF. The second is the complete polarimetric difference matrix method. This method is obtained by a transformation considering neighbor polarimetric covariance matrices [33]. It is denoted as the CP method. The third is the reflection symmetry detector [11], indicated as the RS detector.

It is well known that ship detectors will inevitably produce blurring. When the two targets are close, the detection results of two separated targets can merge into a single blob. To avoid this, we adopted a density-based algorithm to discover erring clusters in large spatial databases with noise (DBSCAN [52]). DBSCAN has two parameters, MinPts, and $\varepsilon$ : MinPts, the minimum number of points in a circle with a radius of $\varepsilon$. Additionally, DBSCAN shows an easier way to ships and false alarms. The related MATLAB codes can be found from https://www.mathworks.com/matlabcentral/fileexchange/5912 3-density-ratio-based-clustering.
For the RADARSAT-2 dataset, the PFA is set to $1 \mathrm{e}-6$, the same as in [48]. Each patch (block) is $500 \times 500$, and the simple truncation threshold is set to 30 . In the DBSCAN algorithm, the distance parameter is $\varepsilon=50$, and the point number MinPts $=10$. The results are listed in Table 1. Fig 13 shows the detection results of different polarimetric detectors clustered by the DBSCAN method ("- 1 " is for false alarms, and other numbers are the detected ships after clustering). For brevity, only figures for NPCM-SQ and PWF are shown.

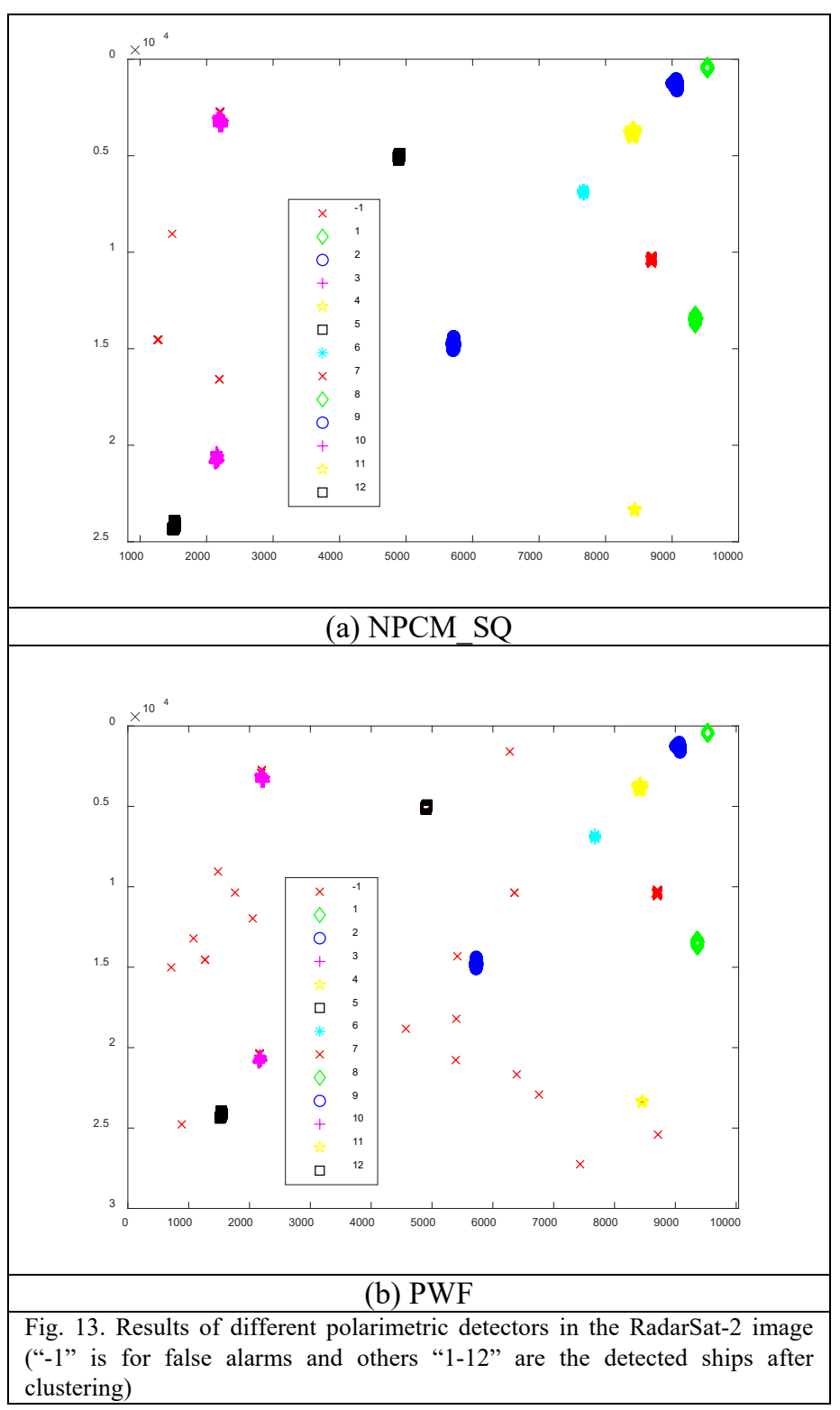

The FOM results show that NPCM-3D achieves the best performance, followed by NPCM-A, NPCM-AS, NPCM-SQ, and PWF after the CFAR processing. Unfortunately, the CP and RS statistical results cannot be modeled as a GGD distribution; therefore, the $\mathrm{CP}$ detector and RS detector are not presented in the FOM table (table 1). The performance results in table 1 are not consistent with the simulation results (where SQ has the best performance). The difference may lie in the fact that each ship's FOM involving many pixels could be counted as one target. 
TABLE I

PERFORMANCES OF ALL DETECTORS IN IN RADARSAT-2

\begin{tabular}{cccccc}
\hline \hline Area & Method & $N_{t d}$ & $N_{f a}$ & $C_{L}$ & $F o M(\%)$ \\
\hline \multirow{4}{*}{ A } & NPCM_SQ & 11 & 20 & 0.78 & 35.48 \\
\cline { 2 - 6 } & NPCM_AS & 11 & 19 & 0.33 & 36.67 \\
\cline { 2 - 6 } & NPCM_A & 11 & 15 & 1.72 & 42.30 \\
\cline { 2 - 6 } & PWF & 11 & 49 & 8.56 & 18.33 \\
\cline { 2 - 6 } & NPCM_3D & 11 & 12 & 3.65 & 47.82 \\
\hline
\end{tabular}

To provide another view where each ship pixel is counted separately, the ROC curves of the real data in RADARSAT-2 are presented in Figure 14. This assumption suggests that more the number of ship pixels detected when the PFA is constant, the better is the detection performance. The results of the measured ROC curves are consistent with the simulation results, where the SQ has the best performance. We can also see that the $3 \mathrm{D}$ reduction results in a better performance than the PWF, which is also consistent with our simulation results. The "NPCM-3D" is worse than other higher dimensional detectors.

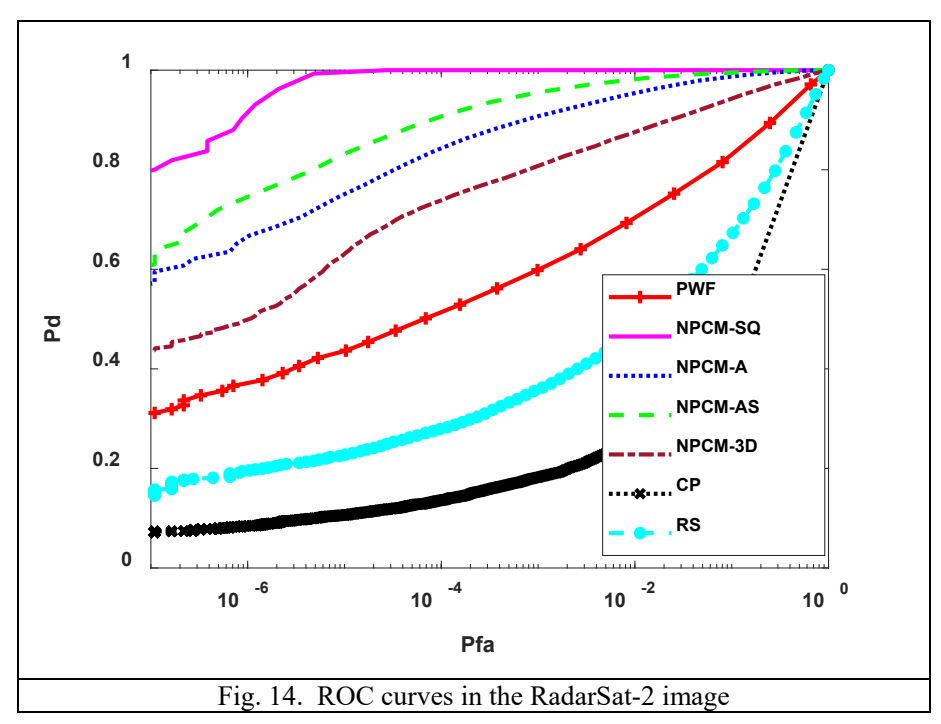

In the AIRSAR dataset, PFA is set to 1e-5 instead of 1e-6 because the background seems to have more variation, and 1e-5 produces more accuracy than 1e-6. The size of each patch (block) is $100 * 100$. The DBSCAN is also applied, and the clustering parameters are the same as Area A. The results are presented in Fig 15, Table 2, and Fig 16. Fig. 15 shows the detection results of different polarimetric detectors after DBSCAN clustering ("-1" is for false alarms and others " $1-12$ " are the detected ships after clustering).

We can see the results from both the table and figures: NPCM_SQ achieves the best performance, and the results are consistent with the simulation results. The "NPCM-3D" almost reaches the same performance as "NPCM-A." It should be noted that in Fig 15(a), one ship is blurring into three at the right of the figure, though all are detected.

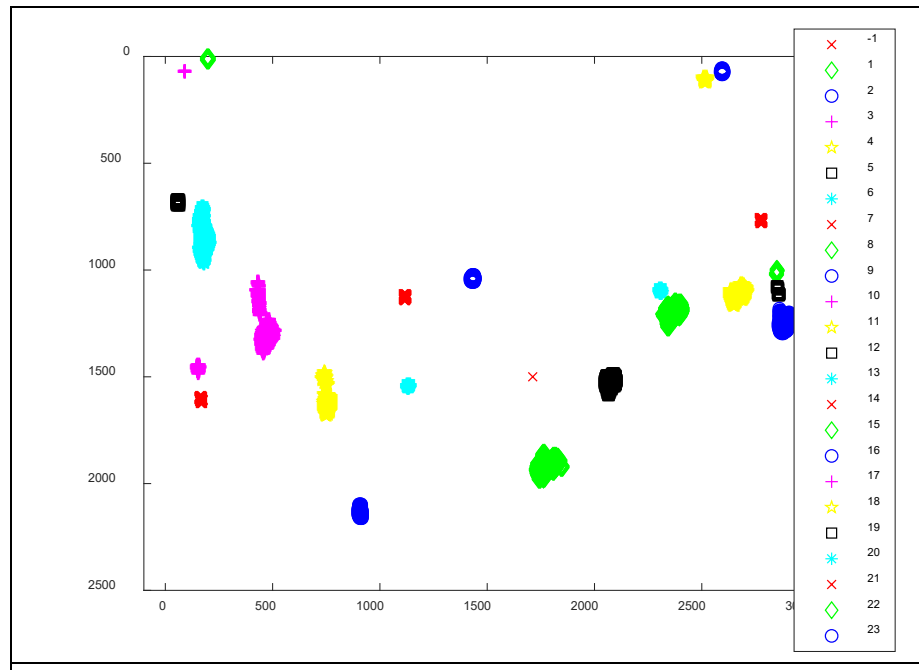

(a) NPCM_SQ

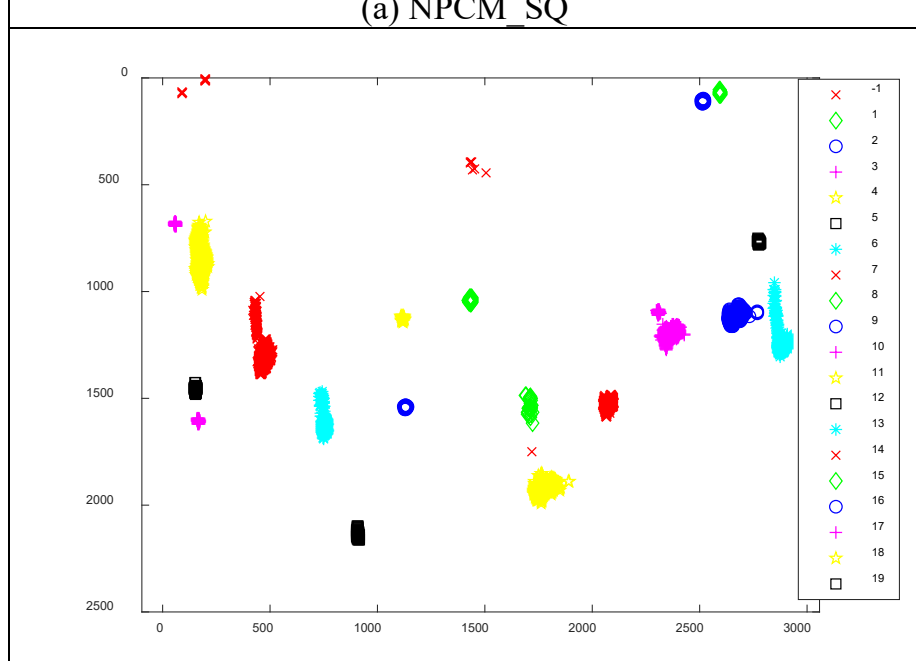

(b) PWF

Fig. 15. Results of different polarimetric detectors in AIRSAR ("-1" is for false alarms and others " $1-12$ " are the detected ships after clustering).

TABLE II

PERFORMANCES OF ALL DETECTORS IN AIRSAR

\begin{tabular}{cccccc}
\hline \hline \multirow{2}{*}{ Area } & \multirow{2}{*}{ Method } & \multirow{2}{*}{$N_{t d}$} & $N_{f a}$ & $C_{L}$ & FoM(\%) \\
\hline \multirow{4}{*}{ B } & NPCM_SQ & 21 & 1 & 12.73 & 91.30 \\
\cline { 2 - 6 } & NPCM_AS & 20 & 4 & 0.69 & 80.00 \\
\cline { 2 - 6 } & NPCM_A & 20 & 8 & 5.33 & 68.97 \\
\cline { 2 - 6 } & PWF & 20 & 15 & 10.79 & 55.56 \\
\cline { 2 - 6 } & NPCM_3D & 20 & 12 & 8.85 & 60.61 \\
\hline
\end{tabular}

Looking at the ROC curves for simulated and real data, it is clear how spatial information is improving the detection dramatically compared to the single-pixel PWF. The ROC also observed that including more pixels in the spatial vectors brings an improvement of performance, although the FOM does not show this for one case. 


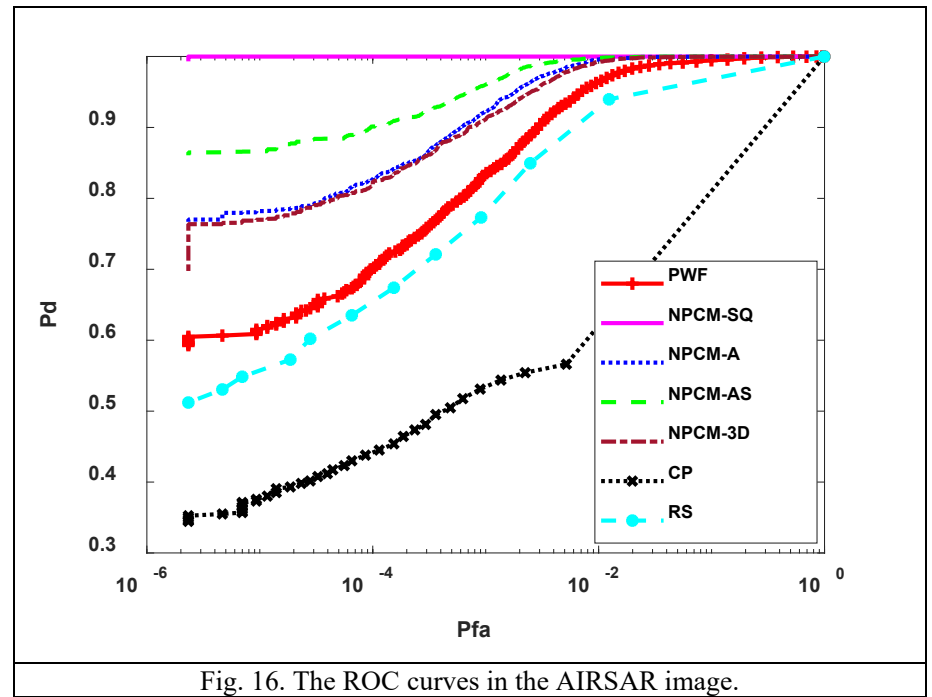

The complete covariance matrix (CP), which also uses spatial information, shows the worst performance. The detection results of the NPCM, PWF, and CP are enlarged and presented in Fig. 17 and Fig. 18. We find the ship pixels from $\mathrm{CP}$ detectors are sparse, which reduces the detection performance. This is probably due to the subtraction of neighborhood pixels representing ships that reduce the total power. The performance of the CP detector is highly dependent on the characteristics of the ship.
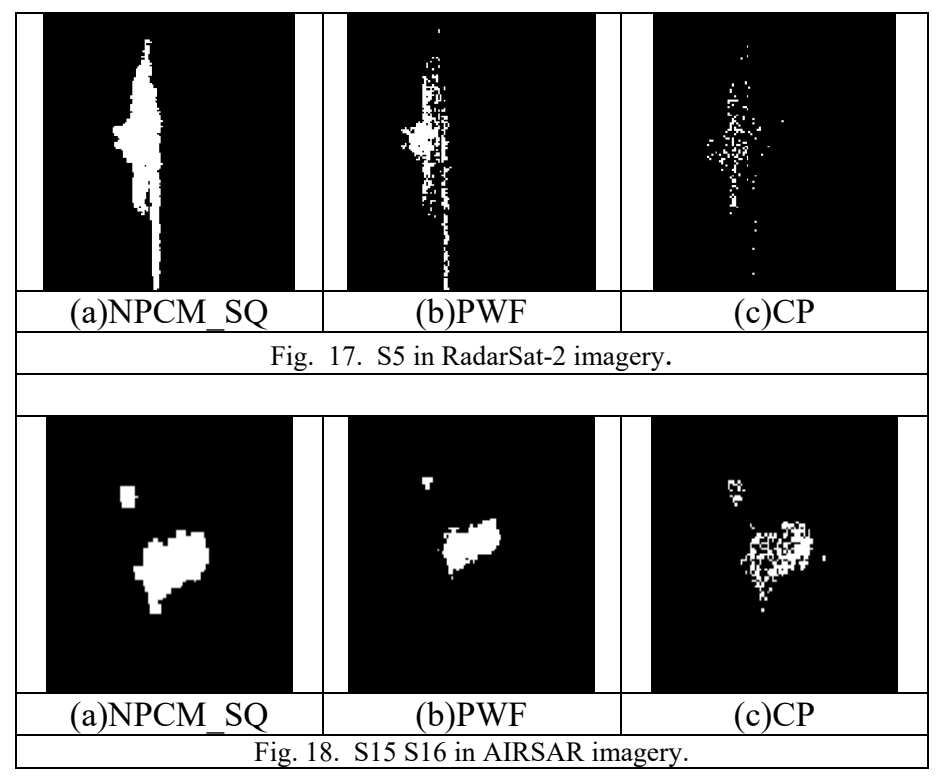

\section{CONCLUSION}

This study proposes a novel polarimetric detector based on the neighborhood spatial correlation to detect small ships in PolSAR images. This idea utilizes the correlation information, including both space and polarization of the neighborhood pixels. As a result, the high dimensional neighborhood polarimetric covariance matrix (NPCM) is established. The traditional detection methods, such as the polarimetric whitening filter (PWF), can be dimensionally extended to whiten the NPCM for ship detection. Owing to the inclusion of spatial information, the novel detector provides better performance than the one using the traditional polarimetric covariance matrix. The PCA form of NPCM almost achieves the best results, although its dimensions are reduced. The theory is validated via simulated and measured data under different sea states and radar platforms.

\section{REFERENCE}

[1] A. Marino, S. R. Cloude, and I. H. Woodhouse, "Detecting depolarized targets using a new geometrical perturbation filter," IEEE Transactions on Geoscience and Remote Sensing., vol. 50, no. 10, pp. 3787-3799, Oct. 2012.

[2] D. J. Crisp, "The state-of-the-art in ship detection in synthetic aperture radar imagery," DSTO, Dept. Defence, Canberra, Australia, Tech. Rep. DSTO-RR-0272, 2004

[3] T. Liu, Z. Yang, A. Marino, G. Gao, and J. Yang, " Robust CFAR Detector Based on Truncated Statistics for Polarimetric Synthetic Aperture Radar," in IEEE Transactions on Geoscience and Remote Sensing. doi: 10.1109/TGRS.2020.2979252

[4] L. M. Novak, M. B. Sechtin and M. J. Cardullo, "Studies of target detection algorithms that use polarimetric radar data," IEEE Transactions on Aerospace and Electronic Systems, vol. 25, no. 2, pp. 150-165, March 1989.

[5] R. Ringrose and N. Harris, "Ship detection using polarimetric SAR data," in Proc. Eur. Space Agency (ESA-SP), vol. 450, Mar. 2000, pp. 687.

[6] J. Chen, Y. Chen, and J. Yang, "Ship detection using polarization crossentropy," IEEE Geosci. Remote Sens. Lett., vol. 6, no. 4, pp. 723-727, Oct. 2009.

[7] M. Sugimoto, K. Ouchi, and Y. Nakamura, "on the novel use of model-based decomposition in SAR polarimetry for target detection on the sea," Remote Sens. Lett., vol. 4, no. 9, pp. 843-852, Sep. 2013.

[8] J. Chen, Y. Chen, and J. Yang, "Ship detection using polarization cross-entropy," IEEE Geosci. Remote Sens. Lett., vol. 6, no. 4, pp. 723-727, Oct. 2009.

[9] R. Touzi, J. Hurley and P. W. Vachon, "Optimization of the Degree of Polarization for Enhanced Ship Detection Using Polarimetric RADARSAT-2," in IEEE Transactions on Geoscience and Remote Sensing, vol. 53, no. 10, pp. 5403-5424, Oct. 2015.

[10] R. Touzi and P.W. Vachon, "RCM Polarimetric SAR for Enhanced Ship Detection and Classification", Canadian Journal of Remote Sensing, Vol. 41, No. 5, pp 473-484, Feb. 2016.

[11] F. Nunziata, M. Migliaccio, and C. E. Brown, "reflection symmetry for polarimetric observation of man-made metallic targets at sea," IEEE J. Ocean. Eng., vol. 37, no. 3, pp. 384-394, Jul. 2012.

[12] L. M. Novak and M. C. Burl, "Optimal speckle reduction in polarimetric SAR imagery," IEEE Transactions on Aerospace and Electronic Systems., vol. 26, no. 2, pp. 293-305, Mar. 1990.

[13] A. Lopes and F. Sery, "Optimal speckle reduction for the product model in multilook polarimetric SAR imagery and the Wishart distribution," IEEE Transactions on Geoscience and Remote Sensing., vol. 35, no. 3, pp. 632-647, May.1997.

[14] G. Liu, S. Huang, and A. Torre, et al., "The multilook polarimetric whitening filter (MPWF) for intensity speckle reduction in polarimetric SAR images," IEEE transactions on geoscience and remote sensing., vol. 36, no. 3, pp. 1016-1020, May. 1998.

[15] R. Touzi, W M Boerner, J S Lee \& E. Lueneburg, "A review of polarimetry in the context of synthetic aperture radar: concepts and information extraction," Canadian Journal of Remote Sensing, vol. 30, no. 3, pp.380-407, 2004.

[16] A. Marino, "A notch filter for ship detection with polarimetric SAR data," IEEE Journal of Selected Topics in Applied Earth Observation and Remote Sensing, vol. 6, no. 10, pp. 1219-1232, 2013.

[17] A. Marino, Sugimoto, Mitsunobu, and Ouchi, et al., "Validating a notch filter for detection of targets at sea with ALOS-PALSAR data: Tokyo Bay," IEEE Journal of Selected Topics in Applied Earth Observation and Remote Sensing, vol. 7, no. 12, pp. 4907-4918, Dec. 2014.

[18] A. Marino and I. Hajnsek, "Statistical tests for a ship detector based on the polarimetric notch filter," IEEE Transactions on Geoscience and Remote Sensing., vol. 53, no. 8, pp. 4578-4595, Aug. 2015.

[19] G. Gao and G. Shi, "CFAR Ship Detection in Nonhomogeneous Sea Clutter Using Polarimetric SAR Data Based on the Notch Filter," IEEE Transactions on Geoscience and Remote Sensing., vol. 55, no. 8, pp. 
4811-4824, Aug. 2017

[20] C. Wang, S. Jiang, H. Zhang, F. Wu, and B. Zhang, "Ship detection for high-resolution SAR images based on feature analysis," IEEE Geosci. Remote Sens. Lett., vol. 11, no. 1, pp. 119-123, Jan. 2014.

[21] X. Leng, K. Ji, K. Yang, and H. Zou, "A bilateral CFAR algorithm for ship detection in SAR images," IEEE Geosci. Remote Sens. Lett., vol. 12, no. 7, pp. 1536-1540, Jul. 2015

[22] J. Ai, X. Yang, F. Zhou, Z. Dong, L. Jia, and H. Yan, "A correlation-based joint CFAR detector using adaptively-truncated statistics in sar imagery," Sensors, vol. 17, no. 4, p. E686, Mar. 2017.

[23] R. Achanta, A. Shaji, K. Smith, A. Lucchi, P. Fua, and S. Süsstrunk, "SLIC superpixels compared to state-of-the-art superpixel methods," IEEE Trans. Pattern Anal. Mach. Intell., vol. 34, no. 11, pp. 2274-2281, Nov. 2012.

[24] W. Yu, Y. Wang, H. Liu, and J. He, "Superpixel-based CFAR target detection for high-resolution SAR images," IEEE Geosci. Remote Sens. Lett., vol. 13, no. 5, pp. 730-734, May 2016.

[25] T. Li, Z. Liu, R. Xie, and L. Ran,"An improved superpixel-level CFAR detection method for ship targets in high-resolution SAR images," IEEE J. Sel. Topics Appl. Earth Observ. Remote Sens., vol. 11, no. 1, pp. 184-194, Jan. 2018.

[26] O. Pappas, A. Achim, and D. Bull, "Superpixel-level CFAR detectors for ship detection in SAR imagery," IEEE Geosci. Remote Sens. Lett., vol. 15, no. 9, pp. 1397-1401, Sep. 2018.

[27] Y. Wang and H. Liu, "PolSAR ship detection based on superpixel-level scattering mechanism distribution features," IEEE Geosci. Remote Sens. Lett., vol. 12, no. 8, pp. 1780-1784, Aug. 2015.

[28] J. He, Y. Wang, H. Liu, and N. Wang, "PolSAR ship detection using local scattering mechanism difference based on regression kernel," IEEE Geosci. Remote Sens. Lett., vol. 14, no. 10, pp. 1725-1729, Oct. 2017.

[29] J. He, Y. Wang, H. Liu, N. Wang and J. Wang, "A Novel Automatic PolSAR Ship Detection Method Based on Superpixel-Level Local Information Measurement," in IEEE Geoscience and Remote Sensing Letters, vol. 15, no. 3, pp. 384-388, March 2018.

[30] X. Cui, Y. Su and S. Chen, "A Saliency Detector for Polarimetric SAR Ship Detection Using Similarity Test," in IEEE Journal of Selected Topics in Applied Earth Observations and Remote Sensing, vol. 12, no. 9, pp. 3423-3433, Sept. 2019, doi: 10.1109/JSTARS.2019.2925833.

[31] Lang, H., Tao, Y., Niu, L. et al. A new scattering similarity based metric for ship detection in polarimetric synthetic aperture radar image. Acta Oceanologica Sinica. 39, 145-150 (2020).

[32] H. Lang, Y. Xi and X. Zhang, "Ship Detection in High-Resolution SAR Images by Clustering Spatially Enhanced Pixel Descriptor," in IEEE Transactions on Geoscience and Remote Sensing, vol. 57, no. 8, pp. 5407-5423, Aug. 2019, doi: 10.1109/TGRS.2019.2899337.

[33] T. Zhang, J. Ji, X. Li, W. Yu and H. Xiong, "Ship Detection From PolSAR Imagery Using the Complete Polarimetric Covariance Difference Matrix," in IEEE Transactions on Geoscience and Remote Sensing, vol. 57, no. 5, pp. 2824-2839, May 2019.

[34] T. Zhang, Z. Yang, H. Gan, D. Xiang, S. Zhu and J. Yang, "PolSAR Ship Detection Using the Joint Polarimetric Information," in IEEE Transactions on Geoscience and Remote Sensing, doi: 10.1109/TGRS.2020.2989425.

[35] K. Jin, et al, " A Patch-to-Pixel Convolutional Neural Network for Small Ship Detection with PolSAR Images," in IEEE Transactions on Geoscience and Remote Sensing. doi: 10.1109/TGRS.2020.2978268

[36] W. Dai, Y. Mao, R. Yuan, Y. Liu, X. Pu, and C. Li, "A Novel Detector Based on Convolution Neural Networks for Multiscale SAR Ship Detection in Complex Background," Sensors, vol. 20, no. 9, p. 2547, Apr. 2020.

[37] Cloude, S.R. and Pottier, E. An entropy based classification scheme for land applications of polarimetric SAR. IEEE Transactions on Geoscience and Remote Sensing. 1997. Vol. 35, No. 1, pp. 68-78. doi: $10.1109 / 36.551935$

[38] Wishart, John. "The Generalised Product Moment Distribution in Samples from a Normal Multivariate Population." Biometrika, vol. 20A, no. $1 / 2$, pp. 32-52, 1928.

[39] Goodman, N. R. "Statistical Analysis Based on a Certain Multivariate Complex Gaussian Distribution (An Introduction)." The Annals of Mathematical Statistics, vol. 34, no. 1, pp. 152-177,1963.

[40] M. Abramowitz, I. A. Stegun, Handbook of Mathematical Functions With Formulas, Graphs, and Mathematical Tables, Dover Publications Inc., NYC, USA, 2001.

[41] T. Liu, J. Zhang, G. Gao, J. Yang and A. Marino, "CFAR Ship Detection in Polarimetric Synthetic Aperture Radar Images Based on Whitening Filter," in IEEE Transactions on Geoscience and Remote Sensing. doi:
10.1109/TGRS.2019.2931353.

[42] F. T. Ulaby, F. Kouyate, B. Brisco and T. H. L. Williams, "Textural Infornation in SAR Images," in IEEE Transactions on Geoscience and Remote Sensing, vol. GE-24, no. 2, pp. 235-245, March $1986 .$.

[43] R. Vaccaro, P. C. Smits and S. G. Dellepiane, "Exploiting spatial correlation features for SAR image analysis," in IEEE Transactions on Geoscience and Remote Sensing, vol. 38, no. 3, pp. 1212-1223, May 2000.

[44] J. Ding, V. Tarokh and Y. Yang, "Bridging AIC and BIC: A New Criterion for Autoregression," in IEEE Transactions on Information Theory, vol. 64, no. 6, pp. 4024-4043, June 2018, doi: 10.1109/TIT.2017.2717599.

[45] A. M. Haimovich and M. Berin, "Eigenanalysis-based space-time adaptive radar: performance analysis," in IEEE Transactions on Aerospace and Electronic Systems, vol. 33, no. 4, pp. 1170-1179, Oct. 1997, doi: $10.1109 / 7.625104$

[46] Armando Marino, A New Target Detector Based on Geometrical Perturbation Filters for Polarimetric Synthetic Aperture Radar (POL-SAR), Springer, ISBN-10: 3642271626, 2012.

[47] E. Ferrentino, F. Nunziata, A. Marino, M. Migliaccio and X. Li, "Detection of Wind Turbines in Intertidal Areas Using SAR Polarimetry," in IEEE Geoscience and Remote Sensing Letters, vol. 16, no. 10, pp. 1516-1520, Oct. 2019, doi: 10.1109/LGRS.2019.2905714

[48] Wei J J, Li P X, Yang J. et al. A new automatic ship detection method using L-band polarimetric SAR imagery[J]. IEEE Journal of Selected Topics in Applied Earth Observations and Remote Sensing, 2014, 7(4): 1383-1393.

[49] P. W. Vachon and J. Wolfe, "C-band cross-polarization wind speed retrieval,"IEEE Geosci. Remote Sens. Lett., vol. 8, no. 3, pp. 456-459, May 2011.

[50]S. Kullback and R. A. Leibler, "On information and sufficiency," Ann. Math. Statist., vol. 22, no. 1, pp. 79-86, 1951.

[51] J. Martín-de-Nicolás, P. Jarabo-Amores, N. del-Rey-Maestre, P. Gómez-del-Hoyo and J. L. Bárcena-Humanes, "Robustness of a Generalized Gamma CFAR ship detector applied to TerraSAR-X and Sentinel-1 images," IEEE EUROCON 2015 - International Conference on Computer as a Tool (EUROCON), Salamanca, 2015, pp. 1-6.

[52] M. Ester, "A Density-based Algorithm for Discover erring Clusters in Large Spatial Databases with Noise," In Proc. 2nd International

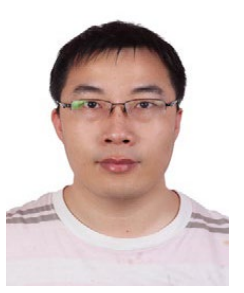

Tao Liu received the B.S. degree in communication engineering and the Ph.D. degree in information and communication engineering, all from the National University of Defense Technology (NUDT), Changsha, China, in 2001 and 2007 respectively.

In 2000, he was the Outstanding Communist Youth League member of China. Since 2007, he has been with the School of Electronic Engineering, Naval University of Engineering, where he is currently a Professor. In 2009 he received the Award of Excellent Doctor Thesis of Chinese Army. Since 2019 he is a committee member in Radar Branch of the Chinese Institute of Electronics (CIE).

He has authored over 50 journal papers and three books. His research interests include statistical theory of radar polarization, polarization information processing, synthetic aperture radar (SAR) automatic target recognition, statistical modeling of SAR image, SAR ship detection, InSAR (interferometric SAR), SAR ground moving target indication and Artificial Intelligence(AI). 


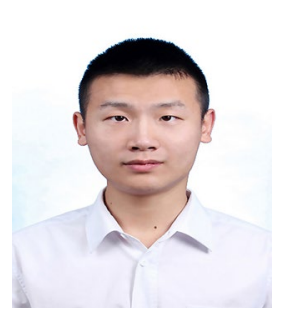

Ziyuan Yang received the B.S. degree in radar engineering from the Naval University of Engineering (NUE), Wuhan, China, in 2019 .Now he is studying for a doctor's degree in information and communication engineering.

His research interests include radar polarization information process and electronic warfare system modeling and SAR ground moving target indication.

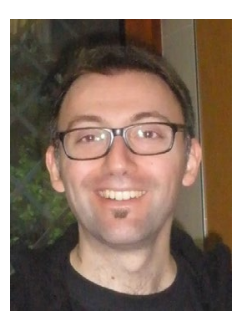

Armando Marino (M'2011) received the M.Sc. degree in telecommunication engineering from the Universita' di Napoli "Federico II," Naples, Italy, in 2006. In 2006, he joined the High Frequency and Radar Systems Department, German Aerospace Centre, Oberpfaffenhofen, Germany, where he developed his M.Sc. thesis. He received the Ph.D. degree in polarimetric SAR interferometry from the School of Geosciences, University of Edinburgh, Edinburgh, U.K., in 2011. From March 2011 to October 2011, he was with the University of Alicante, Institute of Computing Research, Spain. From December 2011 to May 2015, he was a Postdoctoral Researcher and Lecturer with ETH Zurich, Institute of Environmental Engineering, Switzerland. From June 2015, he was a Lecturer with the School of Engineering and Innovation, Open University, Milton Keynes, U.K. Since May 2018 he is a Senior Lecturer (Assistant Professor) at the University of Stirling, Faculty of Natural Sciences, Stirling, UK.

Dr. Marino's Ph.D. thesis received the "Best Ph.D. Thesis 2011 " by the Remote Sensing and Photogrammetry Society and the "Outstanding Ph.D. Thesis" by Springer Verlag, which published the thesis in 2012.

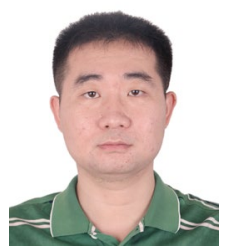

Gui Gao (M'09) received the B.S. in information engineering, the M.S. and Ph.D. degrees in remote sensing information processing from National University of Defense Technology (NUDT), Changsha, China, in 2002, 2003 and 2007, respectively.

From 2007, he joined the Faculty of Information Engineering, School of Electronic Science and Engineering, NUDT, as an associate professor. From 2017, he was with Faculty of Geosciences and Environmental Engineering, Southwest Jiaotong University, Chengdu, China, where he is currently a professor. He is also a guest professor of the College of Traffic Engineering, Hunan University of Technology, Zhuzhou, China. He has authored over 100 journal and conference papers and has written four books and an English chapter. His current research interests include radar signal processing, InSAR (interferometric SAR), target detection, marine environment, and SAR GMTI (ground moving target indication).
Dr. Gao is a member of the IEEE Geoscience and Remote Sensing Society, the Applied Computational Electromagnetics Society, and a senior member of the Chinese Institute of Electronics (CIE), and a Dominant Member of Young Scientist Forum of CIE. He received the Award of Excellent Master Thesis of Hunan Province in 2006, the Award of Excellent Doctor Thesis of Chinese Army in 2008, Awards of Outstanding Young People in NUDT and Hunan Province of China in 2014 and 2016, a first Class Prize of Chinese Military Science and Technology Progress Award, and the Award of Natural Science in Hunan Province. He was also selected as Young Talents of Hunan in 2016 and supported by the Excellent Young People Science Foundation of the National Natural Science Foundation of China. He is the Leader Guest Editor of the International Journal of Antenna and Propagation, the Guest Editor of the Remote Sensing, the Associate Editor and Leader Guest Editor of the IEEE Journal of Selected Topics in Applied Earth Observations and Remote Sensing, and on the Editorial Board of the Chinese Journal of Radars. He was also the Co-Chairman of several conferences in the field of remote sensing. He was the Excellent Reviewer for the journal of Xi' an Jiaotong University in 2013.

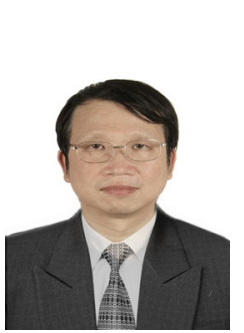

Jian Yang (M'03, SM' 04) received the B.S. and M.S. degrees from Northwestern Polytechnical University, Xian, China, in 1985 and 1990, respectively, and the Ph.D. degree from Niigata University, Niigata, Japan, in 1999. In 1985, he joined the Department of Applied Mathematics, Northwestern Polytechnical University. From 1999 to 2000, he was an Assistant Professor with Niigata University. In April 2000, he joined the Department of Electronic Engineering, Tsinghua University, Beijing, China, and he was promoted to a full Professor in 2002. He has published more than 300 papers and received many awards. His research interesting areas include radar polarimetry, feature extraction, target detection and target classification. 